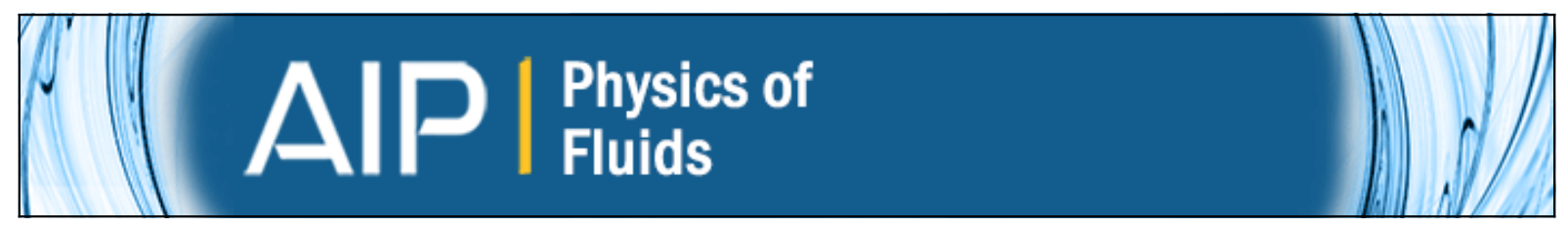

\title{
Streakline-based closed-loop control of a bluff body flow
}

Pablo Roca, Ada Cammilleri, Thomas Duriez, Lionel Mathelin, and Guillermo Artana

Citation: Physics of Fluids 26, 047102 (2014); doi: 10.1063/1.4871716

View online: http://dx.doi.org/10.1063/1.4871716

View Table of Contents: http://scitation.aip.org/content/aip/journal/pof2/26/4?ver=pdfcov

Published by the AIP Publishing

Articles you may be interested in

A direct numerical simulation investigation of the synthetic jet frequency effects on separation control of low-Re flow past an airfoil

Phys. Fluids 27, 055101 (2015); 10.1063/1.4919599

Scalable Implicit Flow Solver for Realistic Wing Simulations with Flow Control

Comput. Sci. Eng. 16, 13 (2014); 10.1109/MCSE.2014.75

Closed-loop controlled vortex-airfoil interactions

Phys. Fluids 18, 046102 (2006); 10.1063/1.2189287

Closed-loop separation control: An analytic approach

Phys. Fluids 18, 043601 (2006); 10.1063/1.2188267

Closed-loop-controlled vortex shedding and vibration of a flexibly supported square cylinder under different schemes

Phys. Fluids 16, 1439 (2004); 10.1063/1.1687413

\section{Did your publisher get}

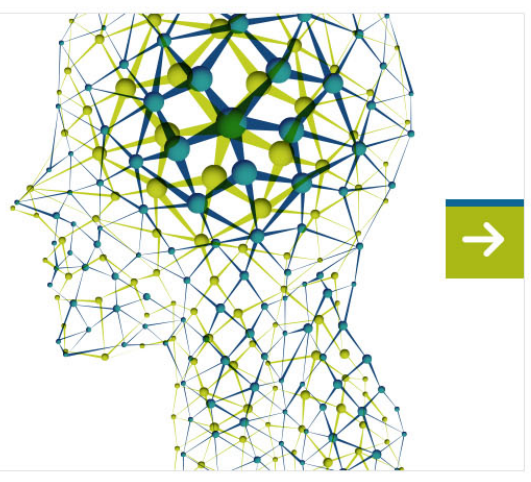




\title{
Streakline-based closed-loop control of a bluff body flow
}

\author{
Pablo Roca, ${ }^{1}$ Ada Cammilleri, ${ }^{1}$ Thomas Duriez, ${ }^{1}$ Lionel Mathelin, ${ }^{2}$ \\ and Guillermo Artana ${ }^{3, a)}$ \\ ${ }^{1}$ Laboratorio de Fluidodinámica, Facultad de Ingeniería, Universidad de Buenos Aires, \\ Buenos Aires, Argentina \\ ${ }^{2}$ LIMSI, CNRS, Orsay, France \\ ${ }^{3}$ CONICET, Laboratorio de Fluidodinámica, Facultad de Ingeniería, Universidad de Buenos \\ Aires, Buenos Aires, Argentina
}

(Received 27 September 2013; accepted 1 April 2014; published online 25 April 2014)

\begin{abstract}
A novel closed-loop control methodology is introduced to stabilize a cylinder wake flow based on images of streaklines. Passive scalar tracers are injected upstream the cylinder and their concentration is monitored downstream at certain image sectors of the wake. An AutoRegressive with eXogenous inputs mathematical model is built from these images and a Generalized Predictive Controller algorithm is used to compute the actuation required to stabilize the wake by adding momentum tangentially to the cylinder wall through plasma actuators. The methodology is new and has real-world applications. It is demonstrated on a numerical simulation and the provided results show that good performances are achieved. (c) 2014 AIP Publishing LLC. [http://dx.doi.org/10.1063/1.4871716]
\end{abstract}

\section{INTRODUCTION}

Control of fluid flows is a discipline with a remarkable importance for industry and science. Classical control objectives such as reference value tracking or disturbances rejection can be applied to many configurations, including drag reduction or instability suppression that are of great interest for practical applications.

On one hand, passive control devices aim at controlling the flow state with a given target without requiring an external power source to operate. In this type of control, the action is permanent and is not suitable for a change in the control parameter since they are strongly tight to the original layout of the experiment. This methodology can be found for instance in vortex generators, ${ }^{1-3}$ or splitter plates. ${ }^{4}$ On the other hand, active control requires applying energy for the flow to attain a defined target. This kind of control allows changing the actuation parameters in time to adjust the controller to new conditions in the experiment. Typical examples of actuators involved with this control are pulsed and synthetic jets, ${ }^{2,5}$ moving objects/surface, ${ }^{6}$ or plasma actuators. ${ }^{7-9}$ These devices are most commonly used in an open-loop control configuration which does not involve feedback mechanisms at time scales comparable to characteristic times of the flow nor prediction of the future state of the actuated flow.

In contrast, closed-loop control usually involves the sensor-based measurement or estimation of the instantaneous state of the flow to feed the controller back and adjust the control parameters in real time. This configuration thus involves evaluation of the actuators inputs from the sensor outputs to achieve a desired effect. This kind of control is suitable for applications with limited amount of energy to manipulate the flow and for systems intended to a relatively wide operating envelope, thereby limiting the drop in performance associated with multiple designs.

Inferring a model describing the system, and capable of predicting the response of the flow to the forcing, from observations is crucial to compute the necessary actuation for a given target. There basically exists two strategies to obtain models that attempt to arrange observations in some

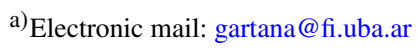


desired state: an approach based on "grey-box" models, say resolving the coherent structures of the flow, and a system identification approach. The former seeks a reduced order model (ROM) relying on a basis deduced from experiments. One of the usual choices of basis has been to consider a Proper Orthogonal Decomposition (POD). The set of equations of the reduced order model is typically obtained by a Galerkin projection of the Navier-Stokes equations onto the POD modes. This procedure leads to a system of ordinary differential equations of as many equations as modes retained in the analysis. However, the retained truncation often neglects high index modes, usually responsible for damping of the physical system, and it may lead to a divergence of the resulting ROM unless a specific treatment is applied. Different efforts have been proposed to stabilize Galerkin-based ROMs. Some of them include additional dissipative models, ${ }^{10}$ or nonlinear Galerkin techniques. ${ }^{11,12}$ Robust techniques based on optimal control strategies have been also proposed for building reduced dynamical models from noisy data, ${ }^{13,14}$ and incomplete knowledge of the actual fluid dynamics, which have demonstrated to be able to extract very accurately low order dynamical systems on the time range on which the data are available. Additional examples of techniques to improve the stability of the reduced order model in fluid flow problems can be found in Refs. 15-21.

Among the advantages of the POD formulation is the possibility to easily define the objective function. When it is desired to minimize fluctuations of the flow, it is possible to directly seek for a minimization of the total energy of the fluctuating part of the flow, which can be determined from the temporal coefficients of the modes (chronos). A restrictive requirement of POD-based ROM approaches is that the data used to generate the model are velocity fields of the flow to be controlled and, from the point of view of field applications, this constitutes a major limitation. Hence, the analysis with this kind of approach has been mostly restricted to numerical benchmarks or experiments in wind tunnels equipped with Particle Image Velocimetry (PIV) systems. A similar limitation applies to other ROM techniques relying on the knowledge of the flow field, such as Balanced Truncation or modal reduction.

Models issued from the second approach, System Identification, are usually empirical black-box models. One of the most popular choices of this methodology is based on an autoregressive relation between current and past system control variables and observables that constitute the state vector. Typical examples are the algorithms based on AutoRegressions with eXogenous inputs (ARX) ${ }^{22-25}$ or AutoRegressions with eXogenous inputs and Moving Average (ARMAX). ${ }^{26}$ Estimation of the parameters of the model is performed using known input-output data retrieved from flow field measurements. The estimation involves minimizing the error norm (typically in the $L_{2}$-sense) between the model output and the actual output from the fluid flow system given the same input data sequence. Shortcomings associated with a system identification strategy such as that described in Ref. 26 comprise effects associated to non-linearities not included in the analysis, a laborious tuning of the parameters of the model and difficulties in the definition of the objective function.

In this work, we propose an improvement upon this last point. Actuation is introduced with the purpose of forcing some characteristic streaklines of the flow to lie into a prescribed region. The objective function to be minimized is defined in terms of the concentration of a tracer injected upstream in the flow, at selected points downstream. To this end, we introduce an image-based approach for active fluid flow control. To the best of our knowledge, this approach is new and it has real-world applications. In particular, the present streaklines-based approach is very appealing in situations where measurement probes cannot be used due to harsh conditions (such as high temperature) or are not available.

Several authors have analyzed the dynamical system associated to a wake flow via the patterns of streaklines passing close to the surface of the body. ${ }^{27-29}$ In these works, the experimentally wellknown concept of streaklines is interpreted as a structure visualizing the unstable manifold of the system. This especially appealing property means that the phase space of the particle dynamics coincides with the configuration space. The phase space structures thus become observable by the naked eye. Therefore, forcing the streaklines to lie in a prescribed region implies, for this kind of flows, to impose a restriction to the dynamical system in the phase space. Additional efforts include closed-loop systems based on the control of the unstable manifolds of Lagrangian separation points as proposed by some authors. ${ }^{30,31}$ However, these control systems were based on wall shear sensors in lieu of instantaneous images of the streaklines. Our approach has similarities with the concept 
of visual servoing techniques. Control strategies based on vision are well established techniques in robotics and automation communities. The method consists in using feedback information provided by a vision sensor observing the system to be controlled. In the field of flow control, only a few efforts have been carried-out along these lines, using the velocity flow fields as input data. ${ }^{32-34}$

In practical applications, the techniques of flow control based on velocity field data often prove impractical. The use of PIV systems outside of a laboratory environment may present some problems related to the use of powerful lasers, dense seeding of tracers required and careful alignment of sophisticated cameras. Further, traditional PIV systems may not be rapid enough for all applications as a consequence of low sampling rates and time required for the processing of the acquired images.

To avoid difficulties associated with the use of PIV, we here present a closed-loop control methodology solely based on the image intensity at a set of probes (selected points of the image of the flow field). Since we do not rely on the velocity field, an input-output-based ARX model of the system is used, in contrast with approaches such as POD or Balanced Truncation which we deem less applicable in practice. The intensity of the image we rely on is proportional to the local concentration of a passive tracer injected upstream.

The prototypical wake produced by a cylinder immersed in a uniform stream is chosen as the physical system to control. The flow is controlled with plasma actuators disposed on the cylinder surface. The present work is focused on a laminar flow at a low Reynolds number (Re, evaluated with the cylinder diameter, free stream velocity and viscosity coefficient) of 235 . This value is close enough to the threshold of appearance of 3D instabilities of the non-actuated flow so that the effects of the 3D instability are weak. Further, a synchronized plasma actuation in the span-wise direction enlarges the range of Reynolds number in which the flow remains 2D. Experiments undertaken with a steady plasma actuation in an open loop system have already shown the authority of this actuator to stabilize the flow at a Reynolds number of $235 .{ }^{35}$ In the present work, we consider the same flow regime and the same actuator configuration as in that article.

We here test the visualization-based control on a numerical benchmark since all system variables are then easily available and this facilitates the evaluation of the control performance. However, the present method can be easily applied in a laboratory environment and beyond, in a real application.

The article is structured as follows. In Sec. II of this article we explain how data were generated using a numerical simulation. The system identification algorithm is presented in Sec. III. In Sec. IV, we propose a Generalized Predictive Controller (GPC) scheme to minimize fluctuations of the wake over a predictive horizon. In Sec. V, the control system is tested and results are discussed. Model validity and robustness under noise in the measurements, tracer injection system, and changes in the inflow conditions are analyzed. Conclusions are drawn in Sec. VI.

\section{PSEUDO-EXPERIMENTAL SET-UP}

We consider a two-dimensional flow around a circular cylinder and the control objective of stabilizing the wake (suppression of the vortex shedding).

Simulations are performed with the code Saturne. ${ }^{36}$ A uniform stream with constant velocity is considered as inlet condition, and the other boundary conditions are indicated in Fig. 1. The Strouhal number of the flow (calculated from the shedding frequency, free-stream velocity, and diameter of cylinder) is close to 0.2. Considering the mesh used and the CourantFriedrichs-Lewy (CFL) condition, the non-dimensional time step of the simulation (based on velocity of the free-stream and cylinder diameter) was set to 0.061. A filament of a passive scalar with a unit constant concentration is injected upstream the cylinder. The concentration of the injected scalar is monitored downstream and a control action is applied at each simulation step. Several probes are symmetrically placed in the wake along the streamwise direction to measure the scalar concentration (see Figs. 2 and 3). Once the non-actuated flow system attains the asymptotic regime, the tracer's concentration at each probe becomes periodic in time (Fig. 4). Note that in a physical experiment, the tracer's concentration at a given point can be associated to the instantaneous gray level of a pixel, or group of pixels, in a single camera snapshot. Unless 


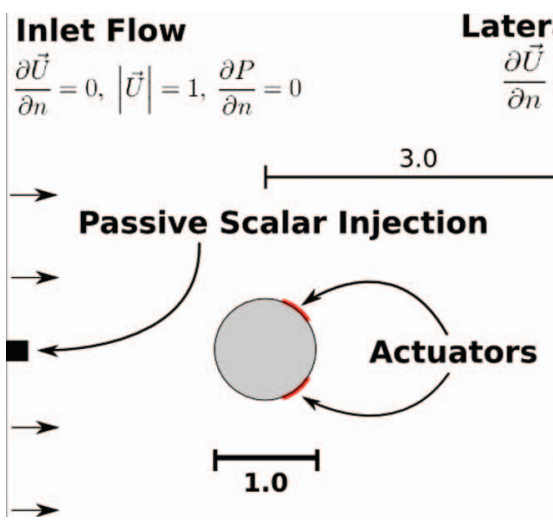

Lateral Symmetry

$$
\frac{\partial \vec{U}}{\partial n}=0, \frac{\partial P}{\partial n}=0
$$

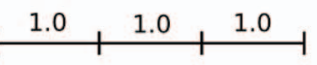

$102 \quad 3 \quad 4$
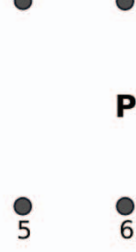

Probes

FIG. 1. Experiment layout. Actuators and scalar concentration probes location $(R e=235$, Simulation domain $27.5 \times 25$ diameters, cylinder vertically centred at 7.5 diameters from the inlet). $\vec{U}$ is velocity, $P$ is pressure, and $n$ the normal direction.

specified, we consider a perfect tracer injection system, perfect sensors, and a spatially uniform, constant in time, velocity at the inlet. In a practical implementation, there can exist fluctuations of the injection system and the cameras used to visualize the flow have errors in the measurements. We will take into account these effects in the closed loop problem by considering a pseudo-random noise introduced in the scalar concentration at the injection point and a pseudo-random noise affecting measurements of the sensors. In some cases, we will also admit that a pseudo-random noise may affect the inlet conditions and that the inlet velocity is altered in time. Two actuators are set on the cylinder surface and add momentum tangentially to the surface. The set-up of the simulation considers plasma actuators ${ }^{37}$ with simultaneous and equal momentum injection over an arc length of about $5 \%$ of the cylinder circumference (Fig. 1). The actuators are located close to the separation line with the goal that the momentum injection produced by actuation promotes the postponement of the separation of the boundary layer from cylinder surface. Previous studies have shown that, with this actuator configuration, a steady forcing of amplitude high enough is able to stabilize the wake flow. ${ }^{35}$ We here propose to stabilize the wake flow with a time varying forcing of much lower amplitude. The actuator is simulated as in Refs. 35 and 38 as an imposed boundary condition in a localized region of the surface of the cylinder. We identify the amplitude of actuation with the value of the tangential velocity at that location at the surface of the cylinder. The control system we propose builds upon the two actuators - that force the flow simultaneously, in phase, and with the same amplitude - and upon the measurements obtained from eight probes. The system identification algorithm that we describe in Sec. III enables to derive a model that links the amplitude of the actuation and the value of scalar concentration at each probe. The control algorithm we consider in this work determines the command required at each time step based on the previous values of the amplitude of actuation, on the scalar concentration at the probes measured in the past, and on the future measurements predicted by the model within a time horizon. Consequently, the controller scheme can be classified as a single input multiple output (SIMO) configuration.

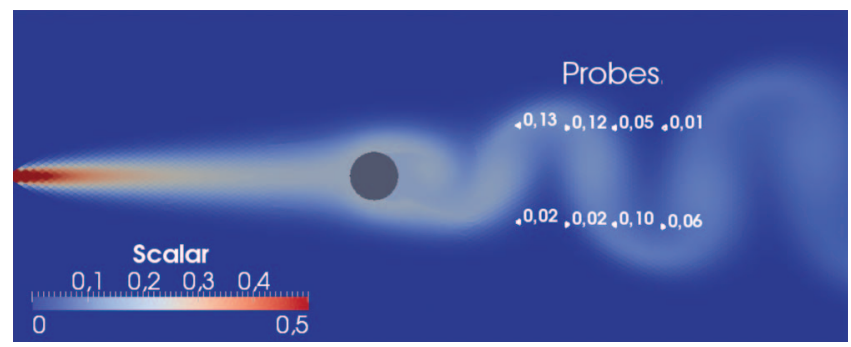

FIG. 2. Instantaneous streakline pattern of the non actuated flow and values of scalar concentration at the different probes. 


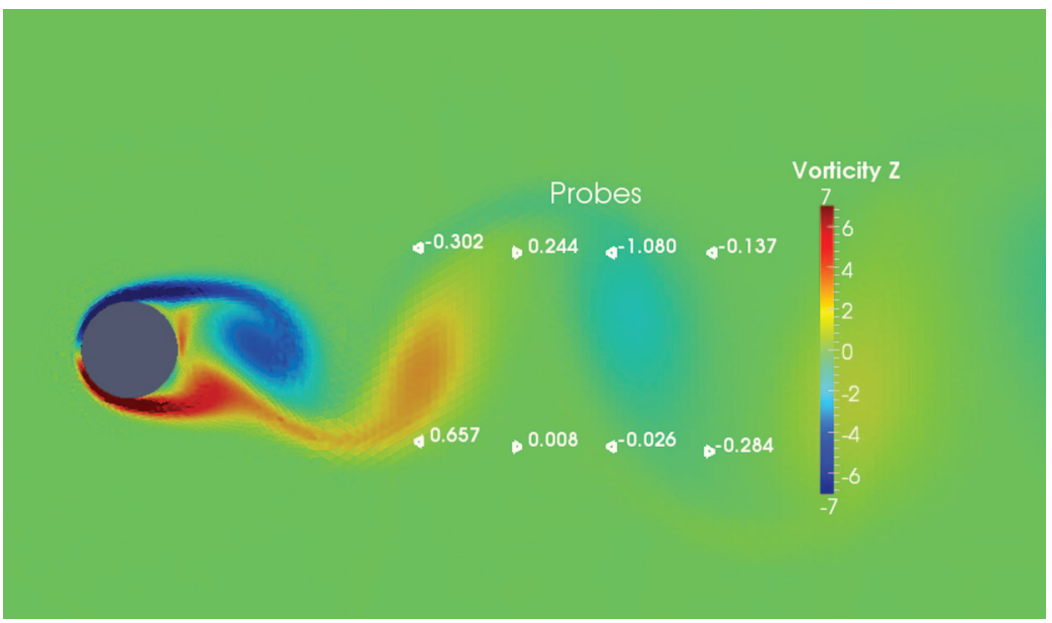

FIG. 3. Instantaneous vorticity field of the non actuated flow and vorticity amplitude at probes location.

\section{SYSTEM IDENTIFICATION}

\section{A. An autoregressive model}

To describe the flow dynamics we consider a linear time-invariant model given by

$$
y(k)+\alpha_{1} y(k-1)+\cdots+\alpha_{p} y(k-p)=\beta_{0} u(k)+\beta_{1} u(k-1)+\cdots+\beta_{p} u(k-p),
$$

where $y(k) \in \mathbb{R}^{m}$ is the system state (the output), $u(k) \in \mathbb{R}^{r}$ the actuation (the input), $p \in \mathbb{N}$ the model order, and $k \in \mathbb{N}$ the current time step. We then associate to the input $u$ the value of actuator's amplitude and to the output $y$ the scalar concentration at the different probes positions.

We refer to this system as an ARX model. The model matrix coefficients, $\alpha_{i}, 1 \leq i \leq p$, and $\beta_{i}$, $0 \leq i \leq p$, of size $m \times m$ and $m \times r$, respectively, are the so-called observer Markov parameters and characterize the model. ${ }^{22}$ The dynamics from $u$ to $y$ may contain a delay of $n_{d}$ samples, so that some first coefficients $\beta_{i}$ are zero. This delay may be explicitly reflected in Eq. (1). However, for easier notations and since this does not introduce any bias, no delay is here explicitly introduced.

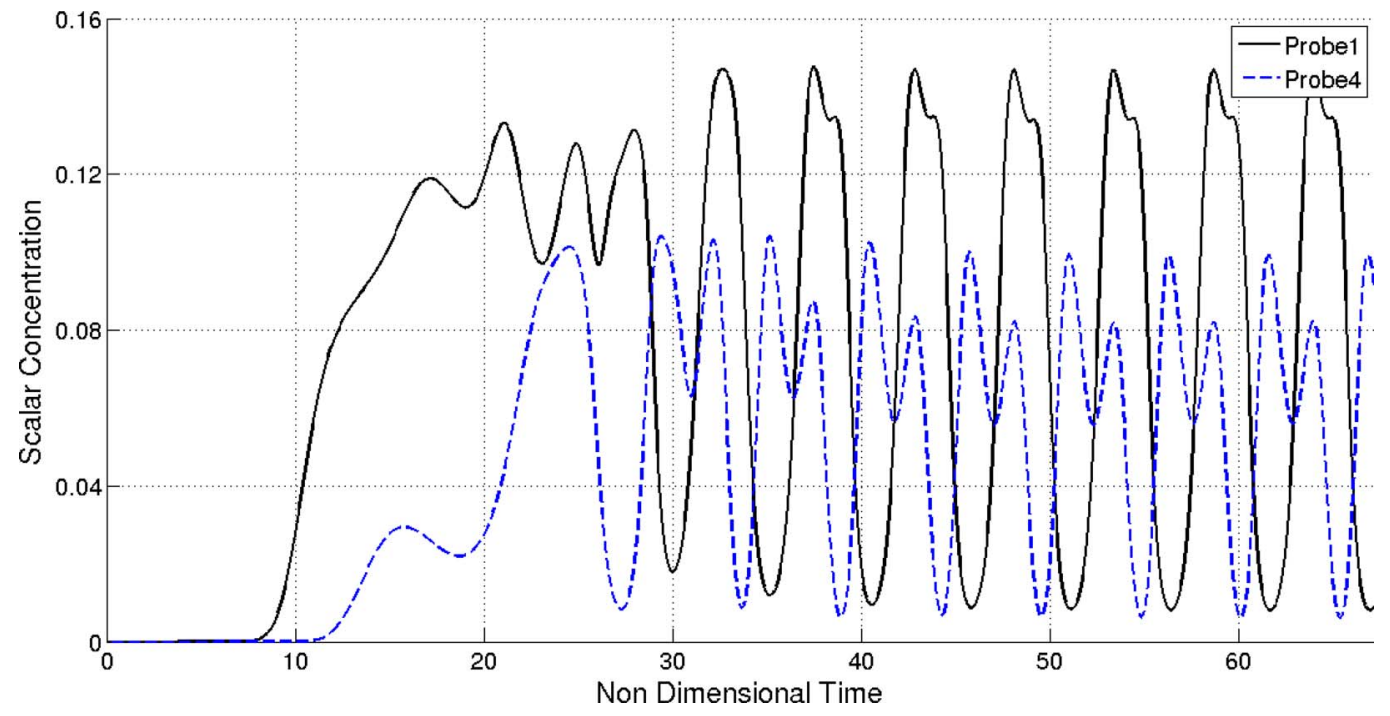

FIG. 4. Time evolution of scalar concentration measured at probes 1 and 4 when starting the simulation. Note the difference in amplitude and the delays in time between the two measures associated to the different positions of the probes. 
Defining the parameters matrix

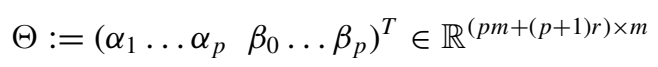

and the regression vector

$$
\varphi(k)=\left(\begin{array}{c}
-y(k-1) \\
\vdots \\
-y(k-p) \\
u(k) \\
\vdots \\
u(k-p)
\end{array}\right) \in \mathbb{R}^{p m+(p+1) r}
$$

Eq. (1) can be written as

$$
y(k)=\Theta^{T} \varphi(k) .
$$

Unfolding matrix $\Theta$ as a vector $\theta$ of size $d:=p m^{2}+(p+1) m r$ which gathers all the coefficients of matrices $\alpha_{i}$ and $\beta_{i}$, and considering $\Xi(k):=\varphi(k) \otimes I_{m}$, where $\otimes$ is the Kronecker product and $I_{m}$ the $m \times m$ identity matrix, Eq. (1) rewrites as ${ }^{39}$

$$
y(k)=\Xi^{T}(k) \theta .
$$

To estimate $\theta$, we consider a training phase where both the actuation and the response of the physical system are known. $N$ training samples are used, together with a least squares criterion, to determine an estimation $\hat{\theta}_{N}^{L S}$ of $\theta$ from

$$
\hat{\theta}_{N}^{L S}=\left(\frac{1}{N} \sum_{k=1}^{N} \Xi(k) \Xi^{T}(k)\right)^{-1} \frac{1}{N} \sum_{k=1}^{N} \Xi(k) y(k) .
$$

When the matrix $\sum_{k=1}^{N} \Xi(k) \Xi^{T}(k)$ is close to being singular, a more effective method may be used to estimate the parameters. ${ }^{39}$ Defining

$$
\begin{aligned}
\Phi^{T} & :=(\Xi(1) \ldots \Xi(N)) \in \mathbb{R}^{d \times N m}, \\
Y^{T} & :=\left(y^{T}(1) \ldots y^{T}(N)\right) \in \mathbb{R}^{1 \times N m},
\end{aligned}
$$

the estimation of $\theta$ is obtained from a QR decomposition of the $N m \times(d+1)$ matrix [ $\Phi Y]$, in which the first $d$ columns are those of the matrix $\Phi$ and the last column is the vector $Y$.

With these definitions, our problem consists in finding $\theta$ which minimizes $\|Y-\Phi \theta\|_{2}^{2}$. We consider a QR-decomposition of matrix $[\Phi Y]=Q R, Q$ being a $N m \times N m$ matrix with orthonormal columns and $R=\left[\begin{array}{c}R_{0} \\ 0\end{array}\right]$ a $N m \times(d+1)$ upper triangular matrix. In particular, $R_{0}=\left[\begin{array}{cc}R_{1} & R_{2} \\ 0 & R_{3}\end{array}\right]$ with $R_{1}$ a $d \times d$ upper triangular matrix, $R_{2}$ a $d \times 1$ vector, and $R_{3}$ a scalar.

$Q$ having orthonormal columns, it comes that $\|Y-\Phi \theta\|_{2}^{2}=\left\|Q^{T}(Y-\Phi \theta)\right\|_{2}^{2}$. Since $Q^{T} \Phi$ contains the first $d$ columns of $R$ and $Q^{T} Y$ is the last column of $R$, it yields

$$
Q^{T} \Phi \theta=\left[\begin{array}{c}
R_{1} \theta \\
0
\end{array}\right] \text { and } Q^{T} Y=\left[\begin{array}{l}
R_{2} \\
R_{3}
\end{array}\right]
$$

So, we obtain $\left\|Q^{T}(Y-\Phi \theta)\right\|_{2}^{2}=\left\|\left[\begin{array}{c}R_{2}-R_{1} \theta \\ R_{3}\end{array}\right]\right\|_{2}^{2}$. When minimizing this quantity and solving the system $R_{1} \hat{\theta}=R_{2}$ we obtain $\hat{\theta}$ the estimation of $\theta$. Also note that $\|Y-\Phi \hat{\theta}\|_{2}^{2}=\left|R_{3}\right|^{2}$.

\section{B. Tuning of parameters}

The training phase was designed to collect input-output instances (Fig. 5) and identify a model of the system. During this phase, the actuators trigger a step pulse and the scalar measurements are 

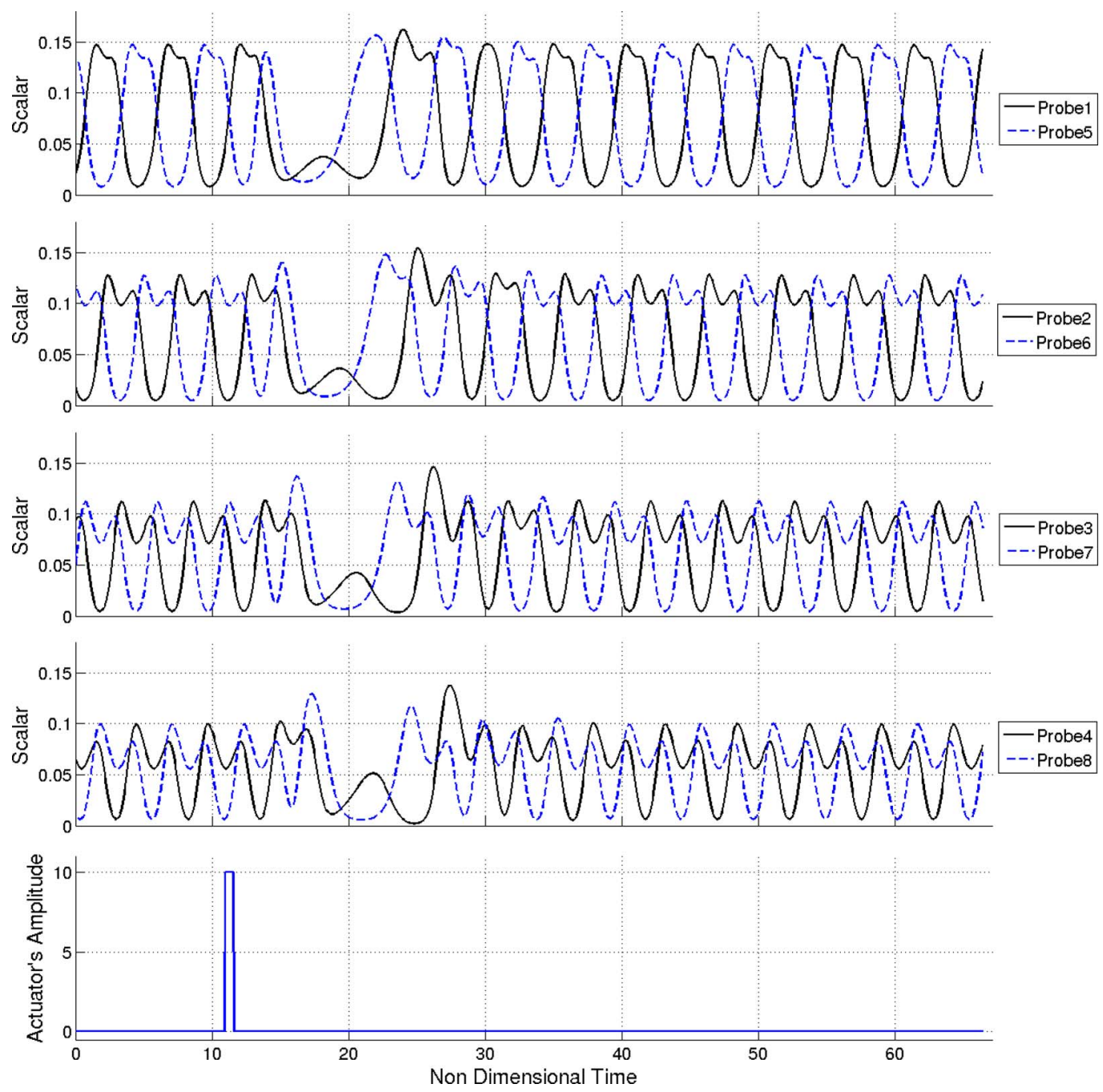

FIG. 5. Training phase of the system. The four upper plots show the scalar concentration evolution in time at the different probes during the training period. The bottom plot shows the pulsed actuation applied simultaneously to each actuator.

used in Eq. (2) to estimate the coefficients. Considering the flow as a nonlinear dynamical system, this forcing step perturbs the orbit in the state space from the attractor. The system then enters a relaxation phase in which the trajectory gradually returns to the orbit around the attractor. Analysis of these transients has been carried-out in some recent works. ${ }^{40}$ When the pulse step is triggered, the scalar concentration in the probes is drastically reduced during a short period of time. This reduction occurs with different delays that depend on the distance of the probe to the cylinder. The number of samples to be considered to train the autoregression model is related to these delays and to the time required for the system to relax to an unperturbed flow dynamics when actuation ceases. ${ }^{26}$

The variables involved in the system identification strategy are the pulse duration of the forcing, the number $N$ of training samples, the sampling rate, the number of probes $m$ and their location, and the horizon of autoregression $p$. In our configuration, the tuning parameters have been set to $p=90$ time steps, $N=1090$, pulse duration of 10 time steps and a non-dimensional sampling step of 0.061 .

\section{Effects of non-linearities}

Interpreting the flow in terms of global modes, in the sense of Ref. 41, stabilization of the wake far above the critical Reynolds number for which the steady flow becomes unstable requires, in 
principle, attenuation of all the unstable global modes. Single-sensor control has been successful in several situations, see Ref. 15 for instance. However, it bears some limitations in terms of control objective and performance as it relies on a very limited amount of information from the system to be controlled. Measurements at different locations within the wake are not simply related by a phase shift and multiple, spatially distributed, sensors offer more flexibility and allow better performance to control the flow. The present image-based approach is hence well suited for a broad class of control objectives since many sensors are available.

To account for the effect of nonlinearities, a nonlinear model is desirable, raising additional issues in identifying such a model. However, linear controllers are sometimes successful in a nonlinear context. Discussions about linear control approaches in nonlinear flows can be found, for example, in Ref. 25. In particular, a strategy based on neural networks has been proposed for a wake flow control problem in a recent work. ${ }^{42}$ Other strategies like adaptive identification algorithms or the stabilization of the flow prior to system identification are also possible. ${ }^{43}$ In agreement with the rationale of other researchers, ${ }^{25}$ for flow control purpose, instead of deriving an accurate system model, our primary goal is to construct a simple input-output data-based system model, which could lead to effective feedback control laws.

When nonlinear interactions between the global modes and the control input are significant, a linearized model developed with the purpose of reducing kinetic energy fluctuations of the flow manages to suppress oscillations of the signal at the sensor locations. ${ }^{41,44}$ Therefore, even when nonlinearities are present, the use of a linear model allows to locally reduce the quantity to be minimized.

We aim at deriving a model to control the dynamics of some streaklines. It reformulates from annihilating all unstable modes to stabilizing solely the modes that significantly drift the flow from its prescribed pattern. Obviously, a physical knowledge of the flow pattern that can be associated to a stabilized flow is required. In the case of the cylinder wake, it is well known that stabilization produces narrower and longer wakes. ${ }^{35}$ Hence, by forcing the wake to exhibit this pattern, the energy associated to the fluctuations might be reduced.

\section{Validation}

To test the validity of the model, we compare tracer's concentration signals, in conditions different from those of the training phase, with the outputs of the identified model (90 time step ahead prediction) $\hat{y}_{i}, i=1, \cdots, 8$. To validate the identified model we generated different data sets at the same Reynolds number changing the flow dynamics by imposing a forcing with the actuators. Two different flow situations were considered. One in which the forcing frequency could not lead to a lock-in with the natural frequency of the flow and another in which this could happen. The objective of these tests is not the control of the flow but rather to assess the predictive capability of our model. Results are analyzed in terms of spectrograms obtained with a Hilbert-Huang spectral analysis. ${ }^{45}$ We test the model with a first data set, labelled data series 1 , associated to a step actuation combined with a sinus function (sinus frequency is 2.25 times the natural frequency of the flow), Fig. 6. We can observe a satisfactory behavior of the model reproducing the main frequencies of the oscillations and not dramatically straying from data (Fig. 8). The system is stable and returns to its equilibrium state once the actuation ceases. However, during the actuation interval, the model predicts signals of tracer concentration with a high frequency component while the actual system does not exhibit these frequencies. This can be related to saturations of the flow dynamics induced by non-linearities that are absent in the identified linear model we consider.

A second data series (data series 2) considers the case when the period of the forcing allows lock-in (Fig. 7). The frequency of the excitation considered is here 1.25 times the frequency of the natural flow and the actuation imposes its dynamics to the vortex shedding. For this data set, the identified model reproduces the signals at the probes correctly without introducing significant spurious frequencies (Fig. 9). 

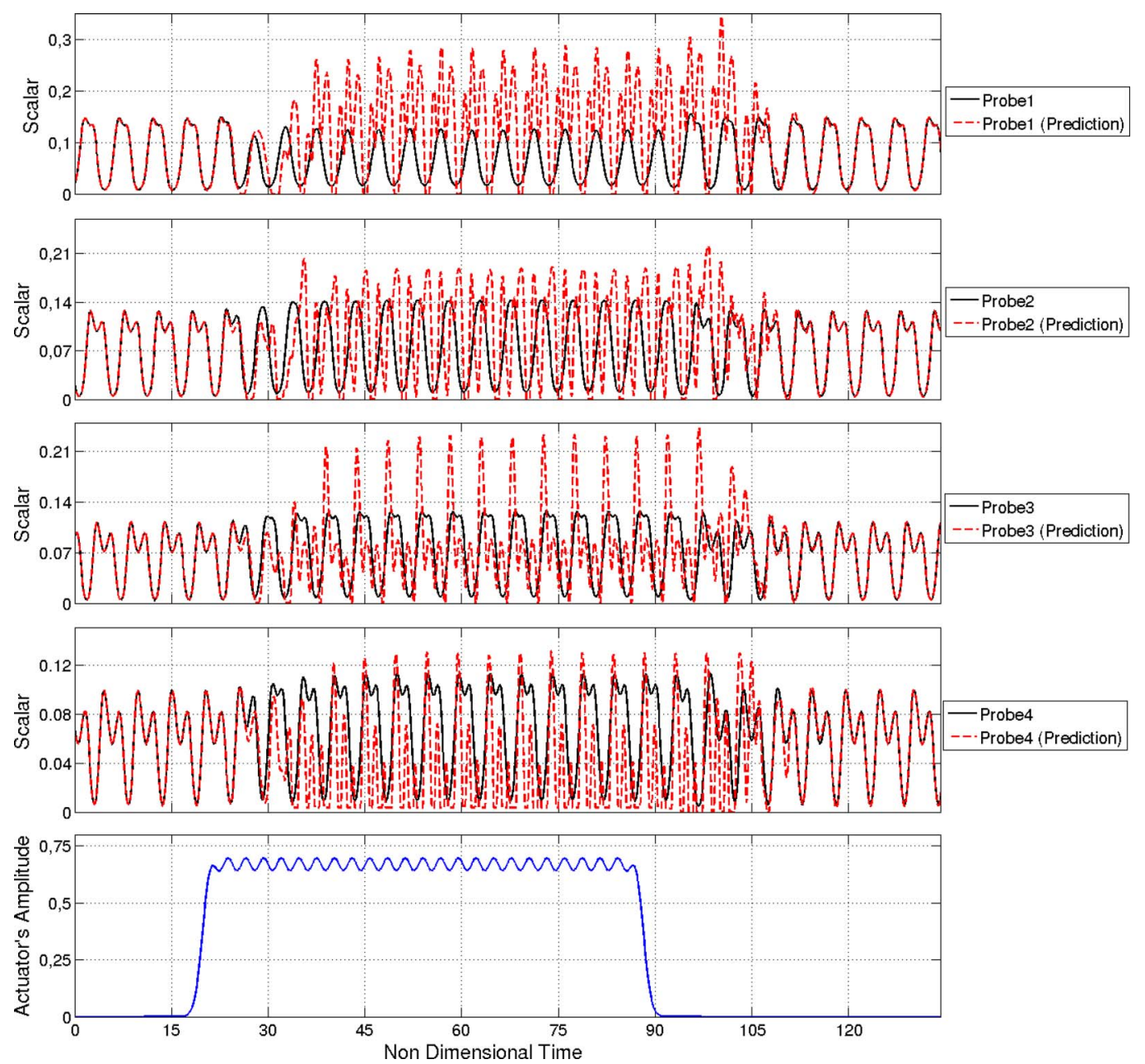

FIG. 6. Model behavior with data series 1. In the upper plots are shown the scalar concentration evolution in time at different probes during the experiment. The bottom plot shows the actuation applied simultaneously to each actuator. The actuation is a step combined with a sinus function having a frequency of 2.25 times the natural frequency of the flow.

\section{CLOSED-LOOP CONTROL ALGORITHM}

Letting $s \in \mathbb{N}$ be the prediction horizon, we define vectors $\hat{y}_{s} \in \mathbb{R}^{s m}, u_{s} \in \mathbb{R}^{s r}$, and $v_{p} \in \mathbb{R}^{p(m+r)}$ according to

$$
\begin{aligned}
\hat{y}_{s}(k):=\left(\begin{array}{c}
\hat{y}(k) \\
\vdots \\
\hat{y}(k+s-1)
\end{array}\right), \quad u_{s}(k):=\left(\begin{array}{c}
u(k) \\
\vdots \\
u(k+s-1),
\end{array}\right), \\
v_{p}(k-p):=\left(\begin{array}{c}
y(k-1) \\
\vdots \\
y(k-p) \\
u(k-1) \\
\vdots \\
u(k-p)
\end{array}\right) .
\end{aligned}
$$



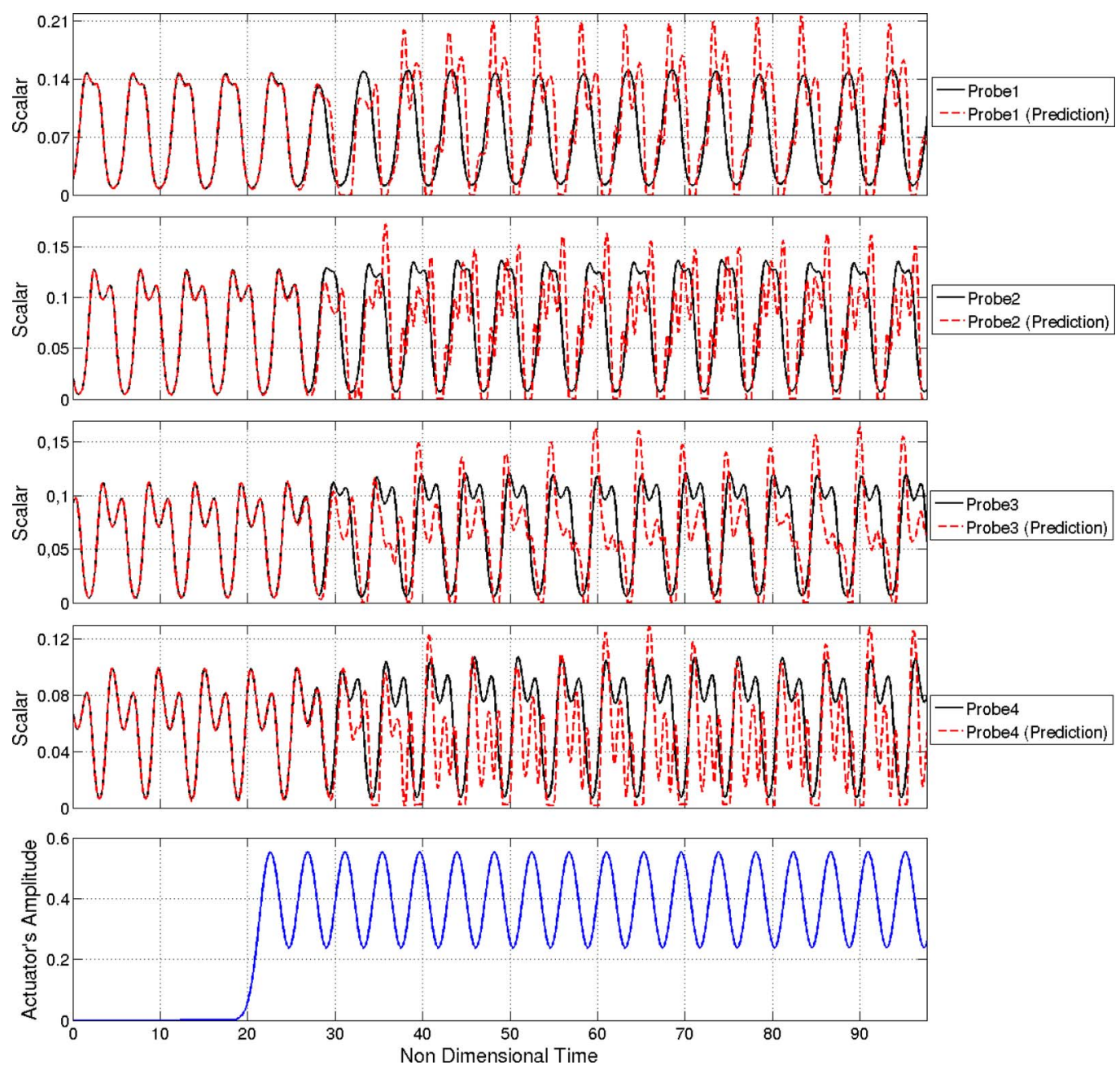

FIG. 7. Model behavior with data series 2 in a lock-in regime. In the upper plots are shown the predictions of the identified model for the scalar concentration evolution in time at different probes. The bottom plot shows the actuation applied simultaneously to each actuator. Actuation is a superposition of a DC component and a sinus function with a frequency 1.25 times the natural frequency of the flow.

From Eq. (1) we can obtain a matrix expression to predict future outputs of the system,

$$
\hat{y}_{s}(k)=\mathcal{T} u_{s}(k)+\Psi v_{p}(k-p),
$$

where $\mathcal{T}$ is a Toeplitz matrix given by

$$
\mathcal{T}:=\left(\begin{array}{ccccc}
\beta_{0} & & & & \\
\beta_{0}^{(1)} & \beta_{0} & & & \\
\beta_{0}^{(2)} & \beta_{0}^{(1)} & \beta_{0} & & \\
\ldots & \ldots & \ldots & \ldots & \ldots \\
\beta_{0}^{(s-1)} & \ldots & \ldots & \beta_{0}^{(1)} & \beta_{0}
\end{array}\right)
$$

with $\beta_{0}, \beta_{0}^{(1)}, \ldots \beta_{0}^{(s-1)}$ known as system Markov parameters, and $\mathcal{T}$ and $\Psi$ matrices of size $m s \times s r$ and $m s \times p(m+r)$, respectively. They are obtained by a series of recursive relations from the model parameters as described in Ref. 22. 

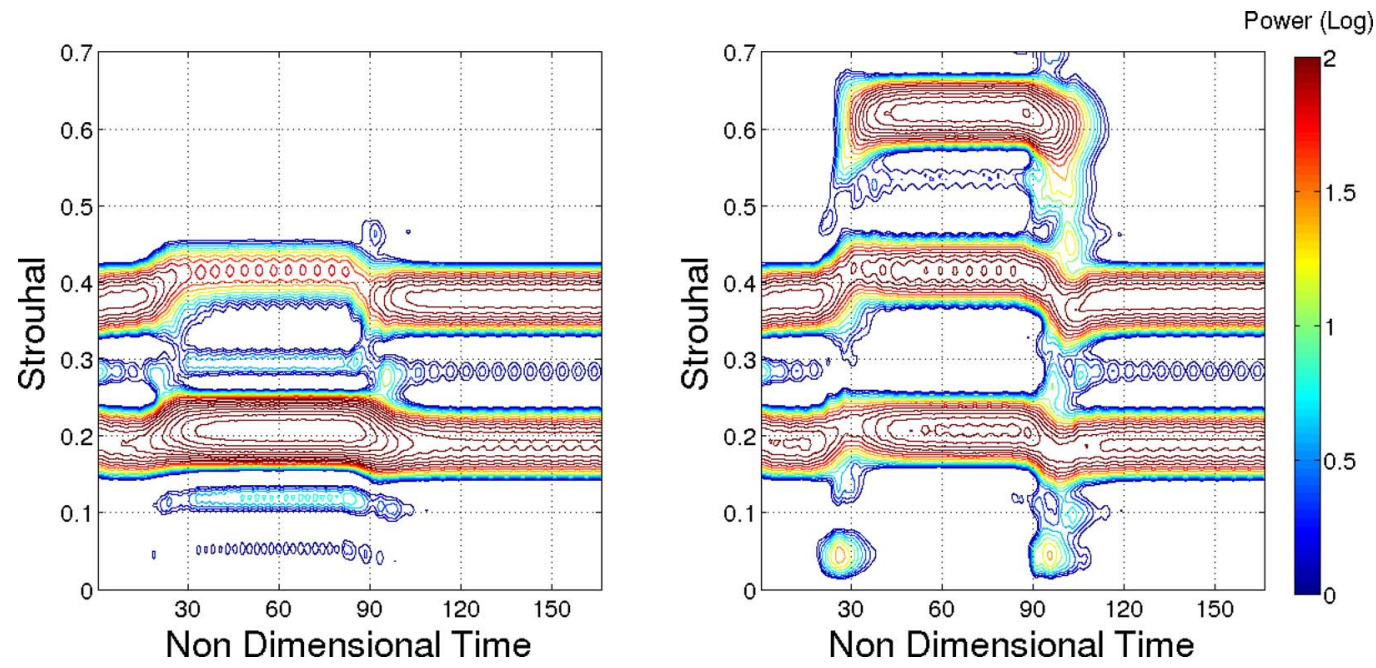

FIG. 8. Comparison of spectrograms of the simulation (left) and predictions of the model built through system identification (right) for the data set of Fig. 6.

The so-called pulse response parameters of the system $\beta_{0}^{(k)}$ are obtained from

$$
\begin{aligned}
& \beta_{0}^{(0)}=\beta_{0}, \\
& \beta_{0}^{(k)}=\beta_{k}+\sum_{i=1}^{k} \alpha_{i} \beta_{0}^{(k-i)}, \quad 1 \leq k \leq p, \\
& \beta_{0}^{(k)}=\sum_{i=1}^{p} \alpha_{i} \beta_{0}^{(k-i)}, \quad k>p .
\end{aligned}
$$

The matrix $\Psi$ is defined as

$$
\Psi:=\left(\begin{array}{cccccccc}
\alpha_{1} & \alpha_{2} & \ldots & \alpha_{p} & \beta_{1} & \beta_{2} & \ldots & \beta_{p} \\
\alpha_{1}^{(1)} & \alpha_{2}^{(1)} & \ldots & \alpha_{p}^{(1)} & \beta_{1}^{(1)} & \beta_{2}^{(1)} & \ldots & \beta_{p}^{(1)} \\
\ldots & \ldots & \ldots & \ldots & \ldots & \ldots & \ldots & \ldots \\
\alpha_{1}^{(s-1)} & \ldots & \ldots & \alpha_{p}^{(s-1)} & \beta_{1}^{(s-1)} & \ldots & \ldots & \beta_{p}^{(s-1)}
\end{array}\right)
$$
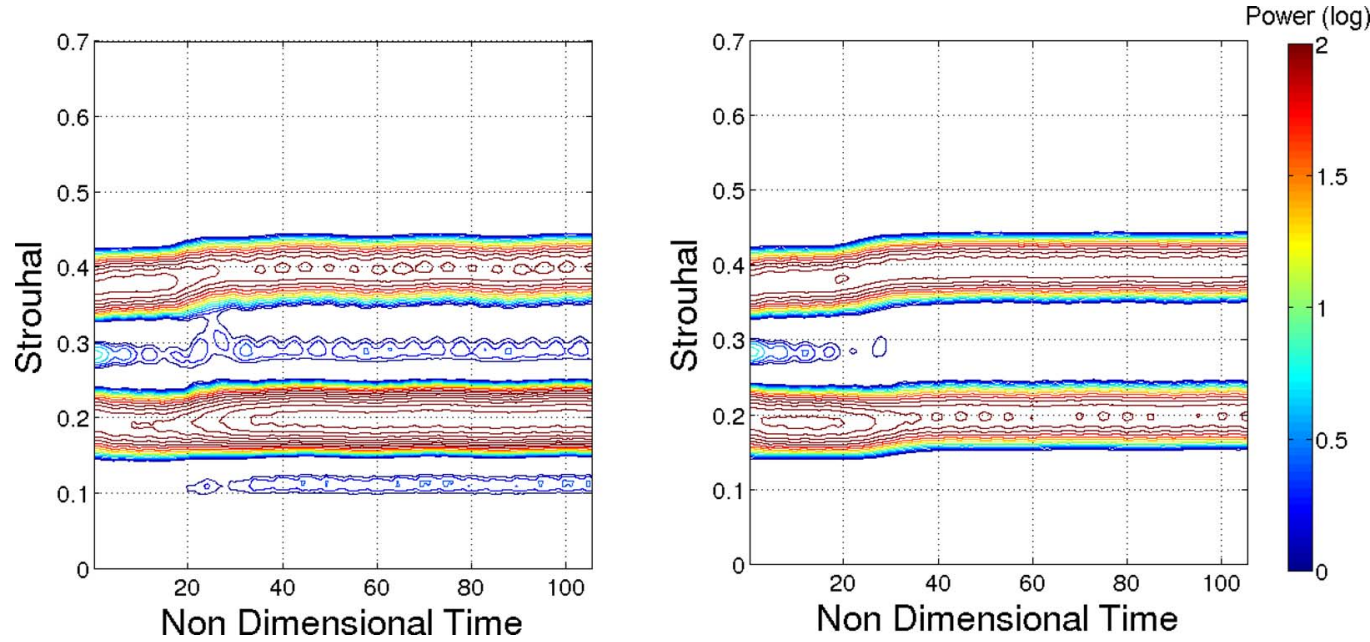

FIG. 9. Comparison of spectrograms of the simulation (left) and predictions of the model built through system identification (right) for the data set of Fig. 7. 
with the following relations for $\alpha_{1}^{k}, \beta_{k}^{j}$, and $\alpha_{k}^{j}$ :

$$
\begin{aligned}
\alpha_{1}^{(0)}= & \alpha_{1}, \\
\alpha_{1}^{(k)}= & \alpha_{k+1}+\sum_{i=1}^{k} \alpha_{i} \alpha_{1}^{(k-i)}, \quad 1 \leq k \leq p-1, \\
\alpha_{1}^{(k)}= & \sum_{i=1}^{k} \alpha_{i} \alpha_{1}^{(k-i)}, \quad k \geq p, \\
\beta_{1}^{(j)}= & \alpha_{1}^{(j-1)} \beta_{1}+\beta_{2}^{(j-1)}, \\
& \vdots \\
\beta_{p-1}^{(j)}= & \alpha_{1}^{(j-1)} \beta_{p-1}+\beta_{p}^{(j-1)}, \\
\beta_{p}^{(j)}= & \alpha_{1}^{(j-1)} \beta_{p}, \\
\alpha_{1}^{(j)}= & \alpha_{1}^{(j-1)} \alpha_{1}+\alpha_{2}^{(j-1)}, \\
& \vdots \\
\alpha_{p-1}^{(j)}= & \alpha_{1}^{(j-1)} \alpha_{p-1}+\alpha_{p}^{(j-1)}, \\
\alpha_{p}^{(j)}= & \alpha_{1}^{(j-1)} \alpha_{p} .
\end{aligned}
$$

We consider a control algorithm based on GPC, ${ }^{46}$ with the objective of decreasing the scalar concentration measured by the sensors, see Ref. 23 for an example of ARX-based GPC. The algorithm generates, at each sampling instant, the future $s$ inputs that minimize the cost function

$$
J=\hat{y}_{s}^{T}(k) Q \hat{y}_{s}(k)+\lambda u_{s}^{T}(k) u_{s}(k),
$$

where $Q$ is a $s m \times s m$ block diagonal matrix with blocks $Q_{i}:=\operatorname{diag}\left(q_{1} \ldots q_{m}\right)$, with $q_{i}$ the weight of sensor $i, 0 \leq q_{i} \leq 1$. The values of these weights are set to unity. The use of different weight values for each probe $i$ allows, for instance, to take into account the downstream diffusion of the vorticity of the tracer.

The parameter $\lambda$ of Eq. (4) penalizes the actuation magnitude. In our experiments, $\lambda$ has always been set to 4 . Note that minimizing the cost function $J$ does not necessarily imply minimizing the kinetic energy of fluctuations. This goal is achieved only by a suitable choice of probes location. The minimization of the cost function only allows to penalize certain, undesirable, streaklines.

We consider a linear control law, $u_{s}(k)=K v_{p}(k-p)$, with $K$ a $s r \times p(m+r)$ matrix containing the controller coefficients.

Applying Lagrange's method to minimize $J$ under the constraint given by Eq. (3), we consider the Lagrangian $L=J+\left\langle l, \hat{y_{s}}-\mathcal{T} u_{s}-\Psi v_{p}\right\rangle$, where $l \in \mathbb{R}^{s m}$ denotes the Lagrange multiplier, and $\langle\cdot, \cdot\rangle$ is a suitable inner product of $\mathbb{R}^{s m}$, yielding the system of optimality:

$$
\begin{aligned}
\hat{y_{s}}-\mathcal{T} u_{s}-\Psi v_{p} & =0, \\
2 Q \hat{y}_{s}+l & =0, \\
2 \lambda u_{s}-\mathcal{T}^{T} l & =0 .
\end{aligned}
$$

One then obtains $K=-\left(\mathcal{T}^{T} Q \mathcal{T}+\lambda I_{s r}\right)^{-1} \mathcal{T}^{T} Q \Psi$, hence

$$
u_{s}(k)=-\left(\mathcal{T}^{T} Q \mathcal{T}+\lambda I_{s r}\right)^{-1} \mathcal{T}^{T} Q \Psi v_{p}(k-p),
$$

where $I_{s r}$ represents the $s r \times s r$ identity matrix.

Since only the actuation value for the current time step $k$ is required, only the first $r$ rows of the product in the first term of Eq. (5) are considered, so that finally

$$
u(k)=\left\{-\left(\mathcal{T}^{T} Q \mathcal{T}+\lambda I_{s r}\right)^{-1}\right\}_{r} \mathcal{T}^{T} Q \Psi v_{p}(k-p) .
$$


Once the actuation $u$ is computed from Eq. (6) and the measurements $y$ are taken, $v_{p}$ is updated and the whole process is repeated at the next time step. The resulting control algorithm is now summarized:

ALGORITHM 1. Proposed control algorithm.
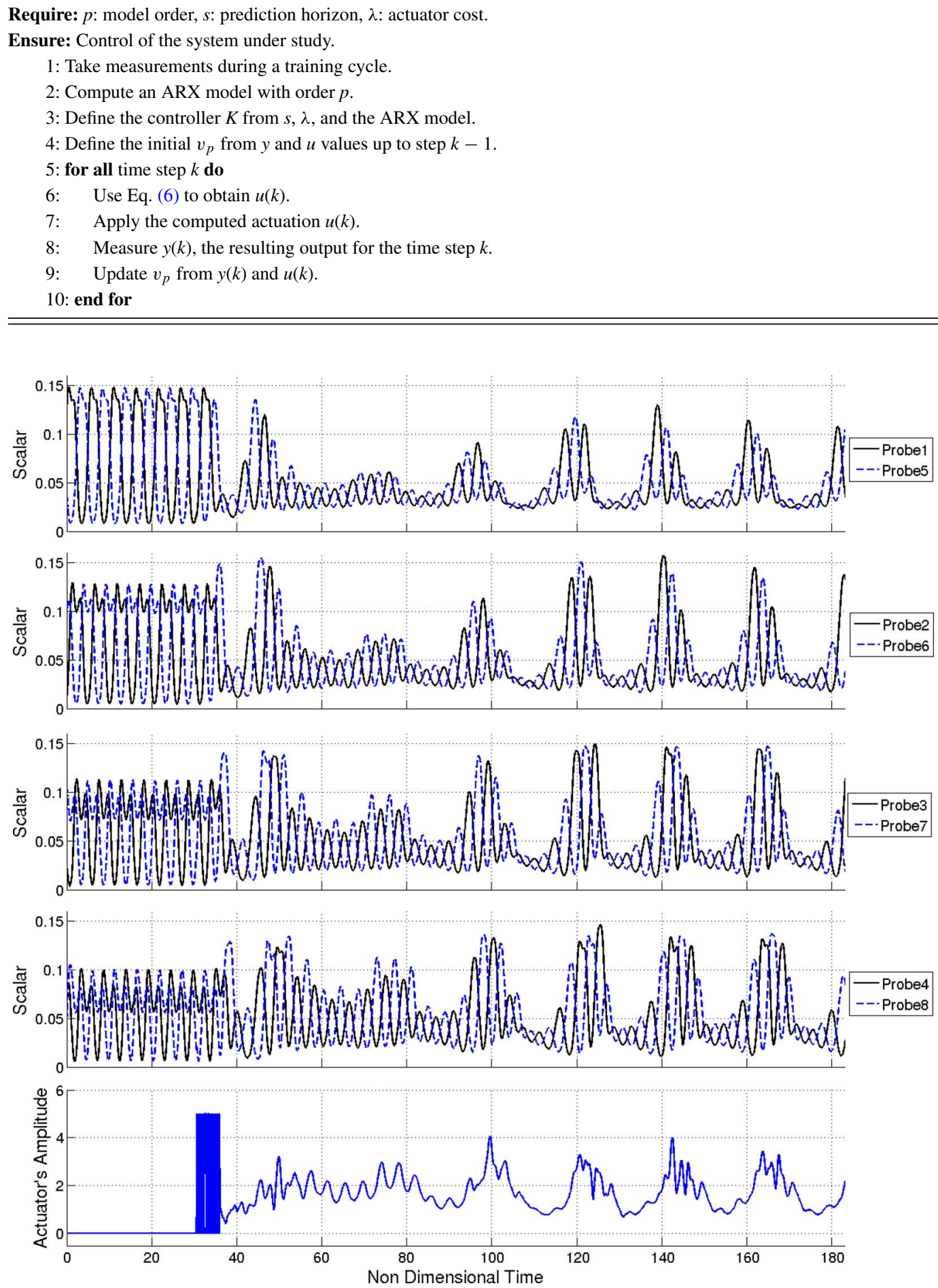

FIG. 10. Closed-loop control results. Scalar concentration at different probes and amplitude of actuation as a function of time. 


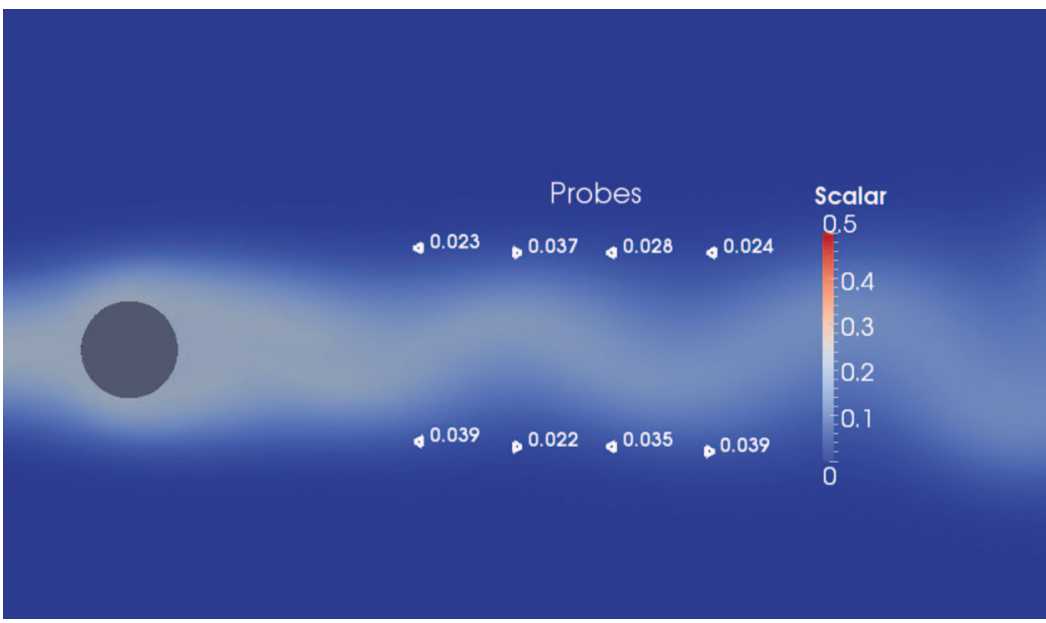

FIG. 11. Instantaneous streaklines and values of scalar concentration at the probes of the controlled wake.

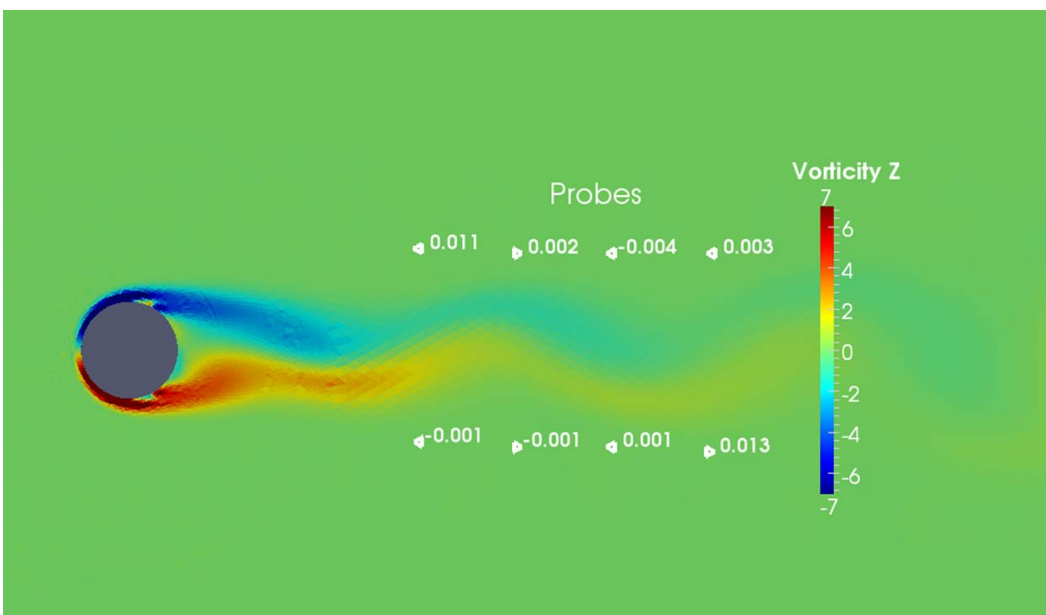

FIG. 12. Instantaneous vorticity field and values at the probes of the controlled flow.

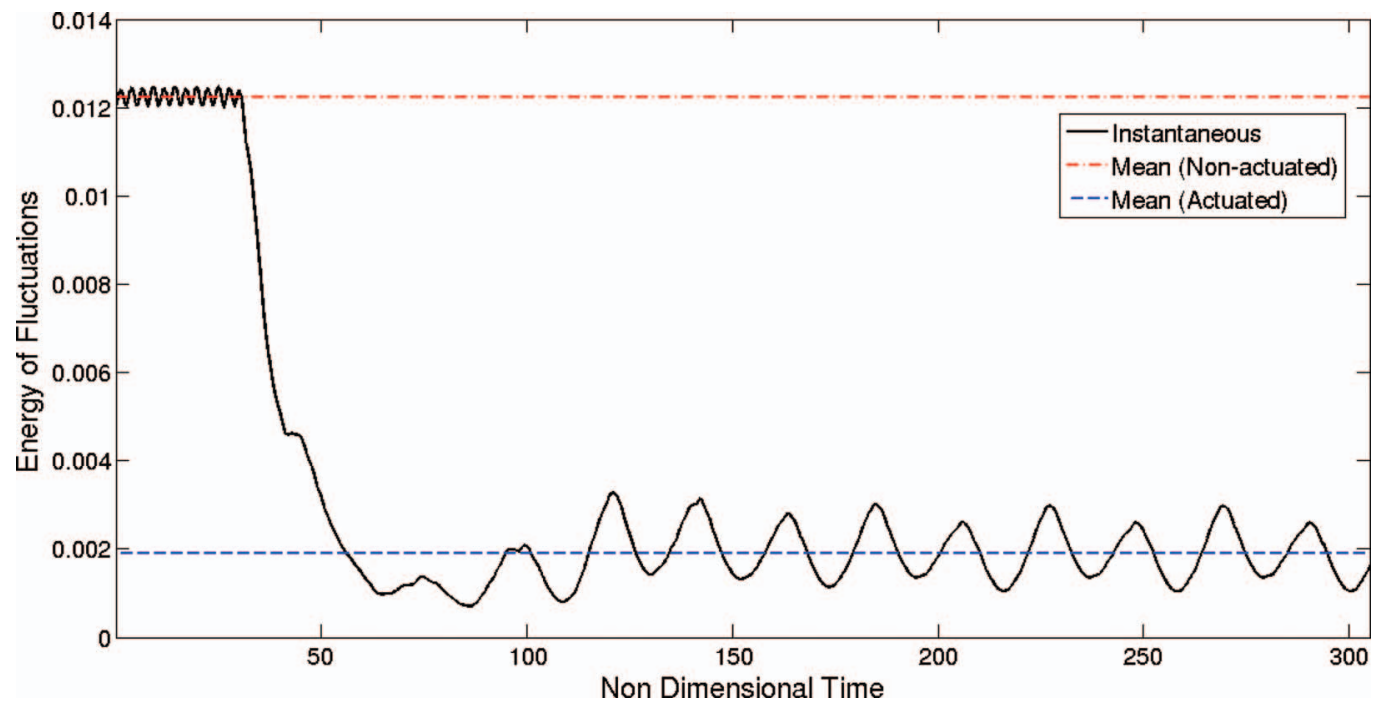

FIG. 13. Instantaneous and time-averaged kinetic energy of fluctuations for the non-actuated and controlled flow. Control is activated at a non-dimensional time of 500 . 


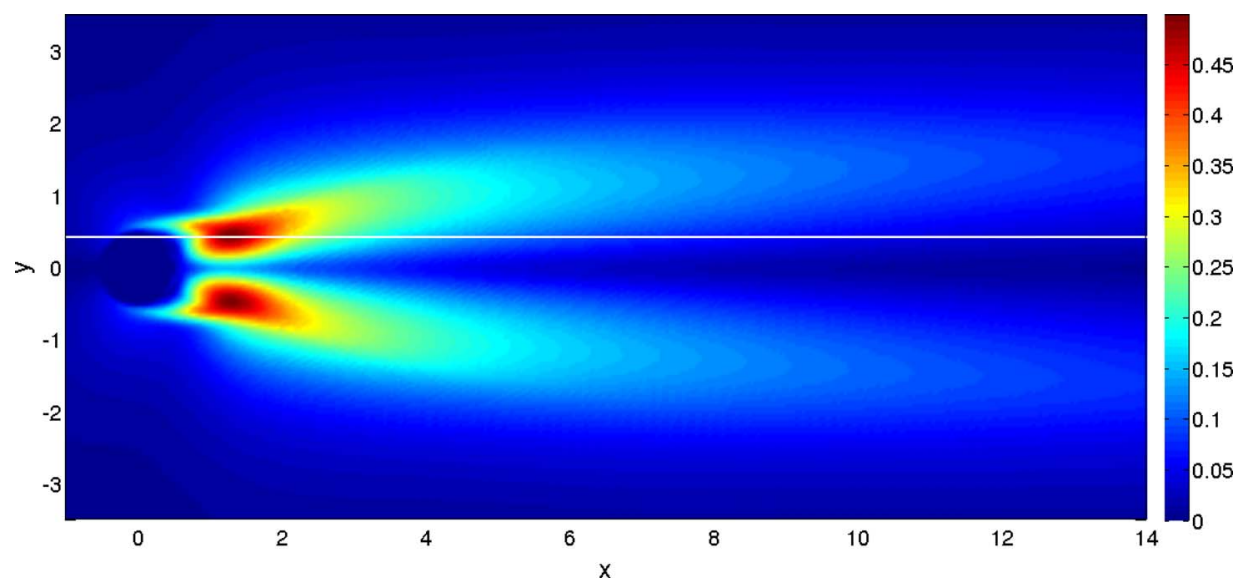

FIG. 14. RMS of the velocity in the stream-wise direction for the non-controlled flow. The horizontal white line at $y=0.415$ is in correspondence with the maximum of the RMS.

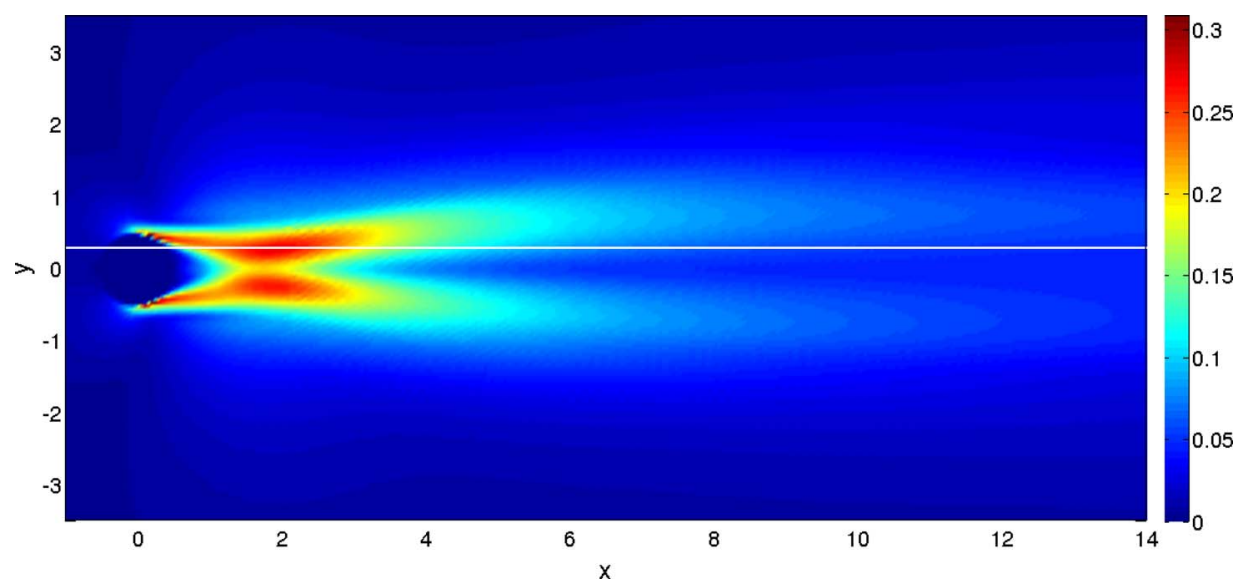

FIG. 15. RMS of the velocity in the stream-wise direction for the controlled flow. The horizontal white line at $y=0.280$ is in correspondence with the maximum of the RMS.

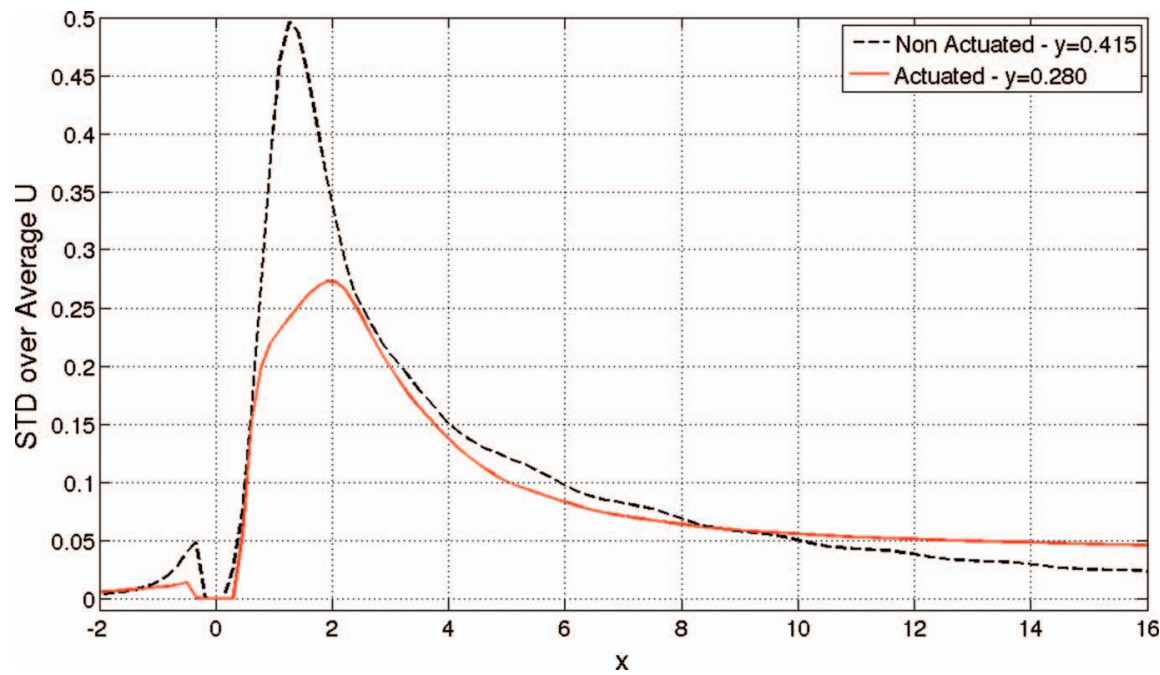

FIG. 16. RMS of the velocity in the streamwise direction along white lines indicated in Figures 14 and 15. 


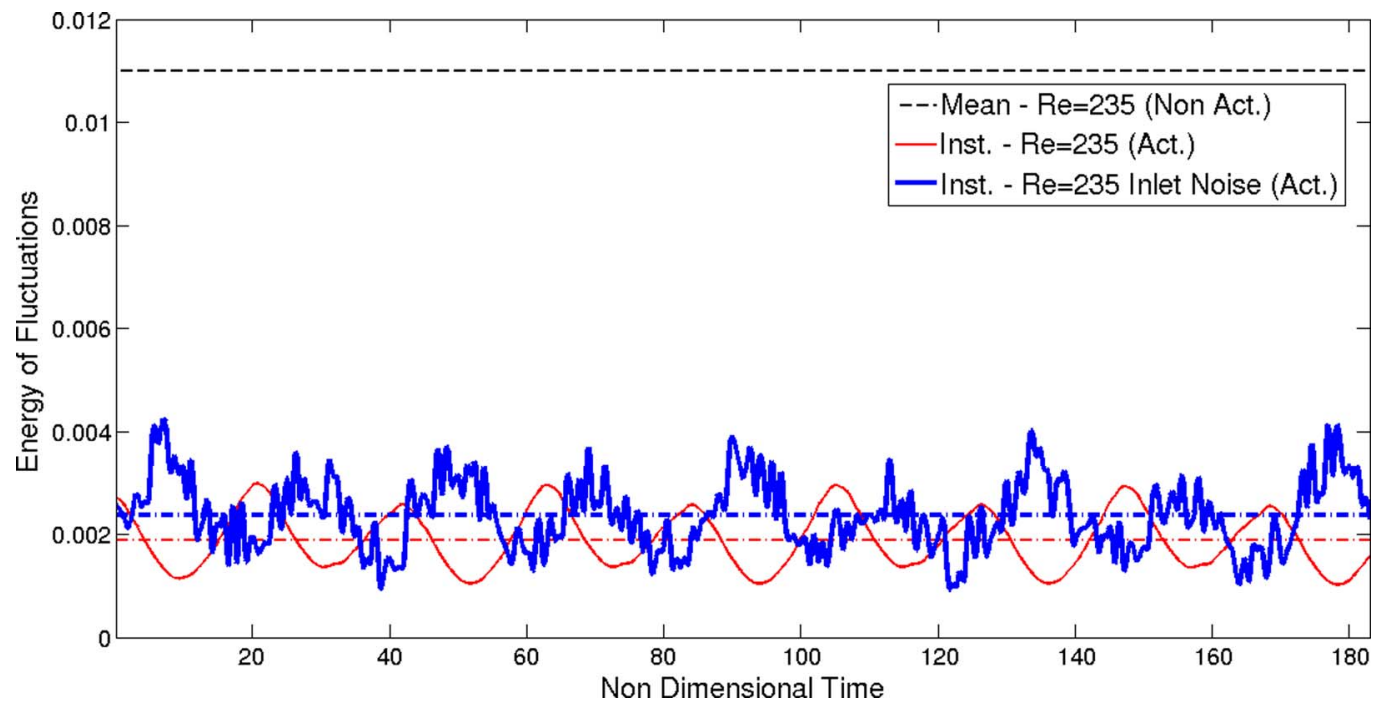

FIG. 17. Instantaneous values and time averages of the kinetic energy of the controlled flow when a pseudo-random noise of $10 \%$ is added to the inlet flow velocity.

\section{CONTROL SYSTEM RESULTS}

Once the control parameters are tuned, the physical model is simulated and the trained controller determines an actuation signal that stabilizes the flow.

In Fig. 10, the clear link between actuation and scalar concentration is shown. As we can see, once the actuation is activated and after a short period of time, the control system stabilizes. A decrease (increase) of scalar concentration detected by the sensors leads to a fast decrease (increase) of the magnitude of actuation. The instantaneous images of streaklines (Fig. 11) and vorticity field (Fig. 12) compared to the non-actuated flow (Figs. 2 and 3) show the stabilization of the wake as a consequence of the forcing and how the wake is controlled.

To further illustrate the stabilization of the wake, we represent in Fig. 13 the energy associated with fluctuations as a function of time for the non-actuated and the actuated flow. As we can see, a reduction of about $85 \%$ in energy is obtained.

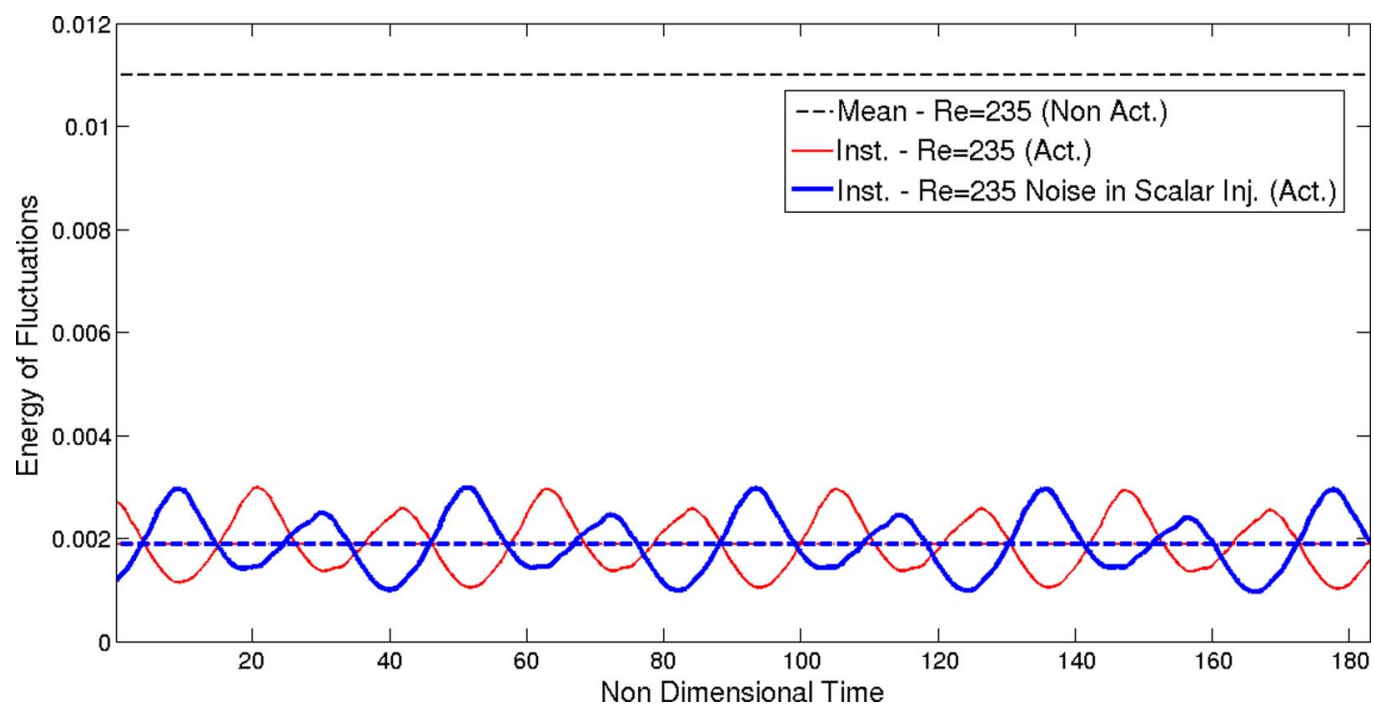

FIG. 18. Instantaneous values and time averages of the kinetic energy of the controlled flow when a pseudo-random noise of $10 \%$ is added to the injection system. 


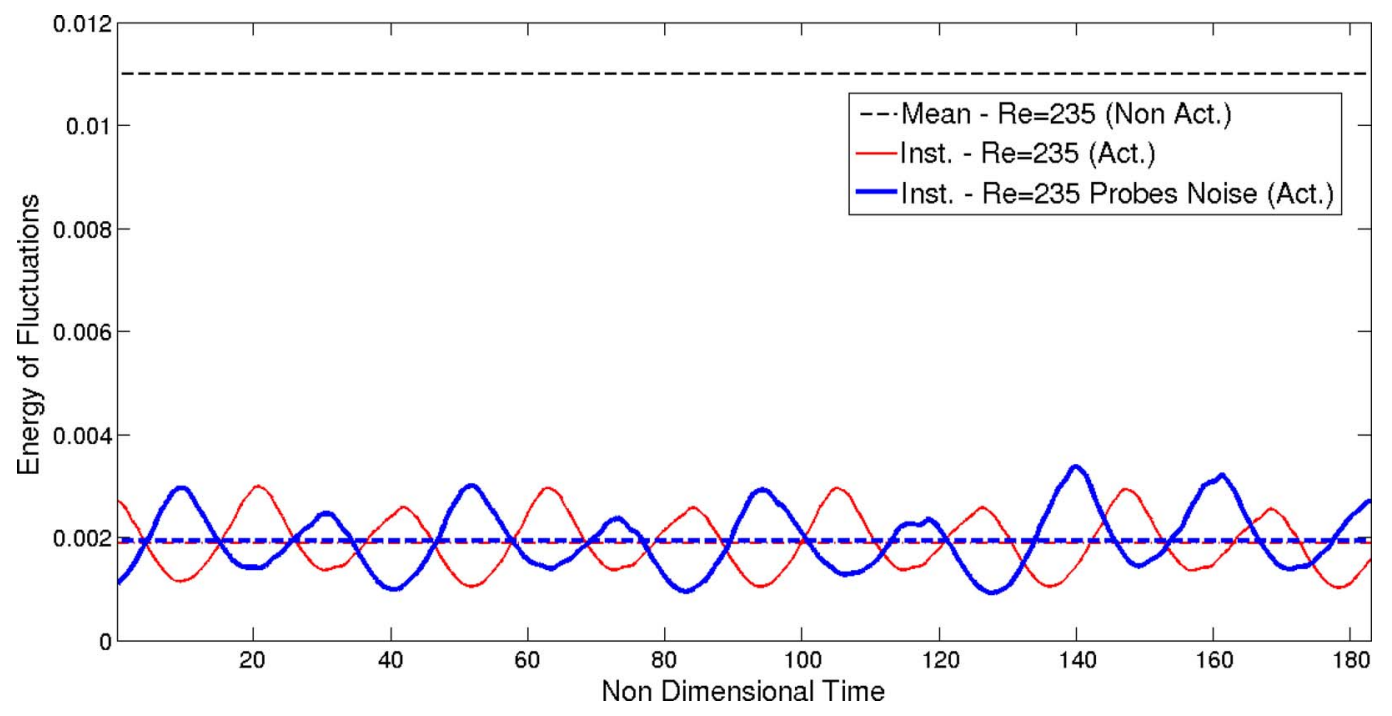

FIG. 19. Instantaneous values and time averages of the kinetic energy of the controlled flow when a pseudo-random noise of $0.1 \%$ is added to the sensors.

The control impact can also be appreciated from the plot of the RMS fields of the velocity in the stream-wise direction (Figs. 14 and 15). As can be seen, the control system forces the wake fluctuations to be concentrated within the narrow path delimited by the probes. We show in Fig. 16 the RMS values along an horizontal line containing the point that exhibits the maximum value of fluctuations (indicated as white lines in Figs. 14 and 15). The decrease of the peak values of the RMS and the shift of this maximum towards downstream positions that we can observe in Fig. 16 indicates the stabilizing effect of the control on the wake. These results are similar to those obtained with a continuous forcing. ${ }^{35}$

The validity of the control algorithm under conditions different from the training configuration is now considered with cases in which a pseudo-random noise of amplitude $10 \%$ affects the uniform inlet flow velocity (Fig. 17), a pseudo-random noise of $10 \%$ affects the concentration injection (Fig. 18), and pseudo-random noise of $0.1 \%$ affects sensor measurements (Fig. 19). In all these cases the behavior of the control system was satisfactory and illustrates its robustness.

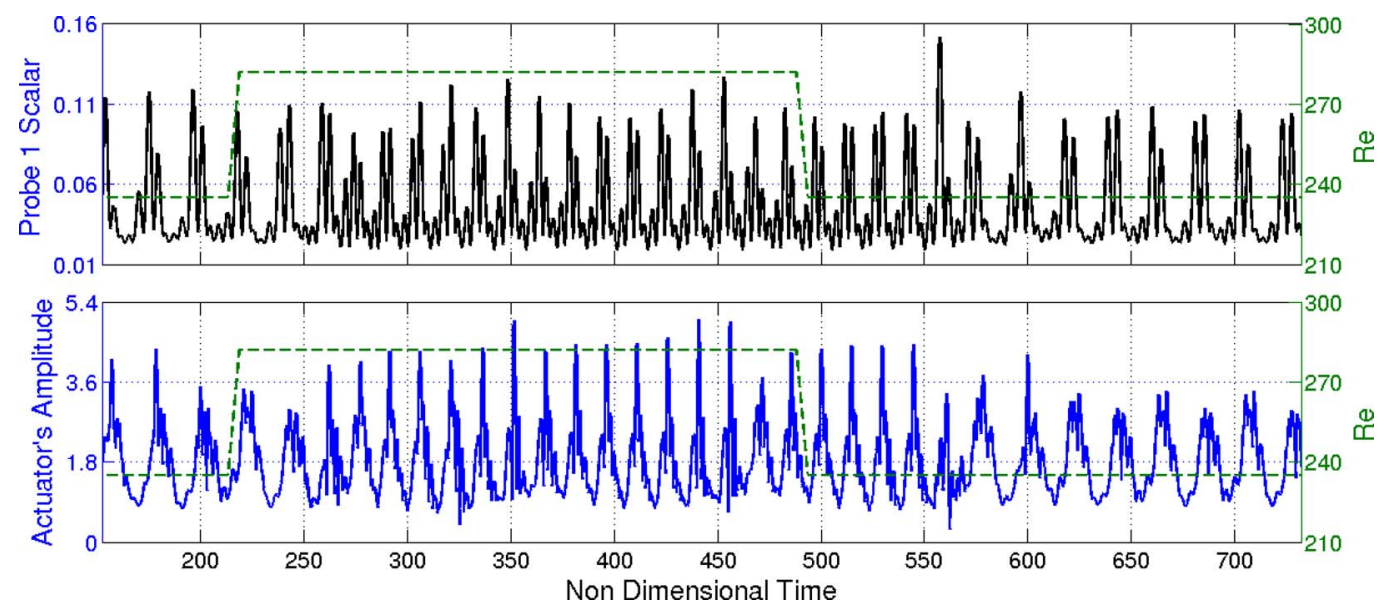

FIG. 20. Changes in the inlet condition. The Reynolds number increases from 235 to 282 and recovers its initial value following a ramp (green-dashed plot). Upper plot: Scalar concentration at probe 1 as a function of time. Lower plot: Instantaneous amplitude of the forcing in each actuator. The control system adapts its command to stabilize the wake to the new inlet condition. 

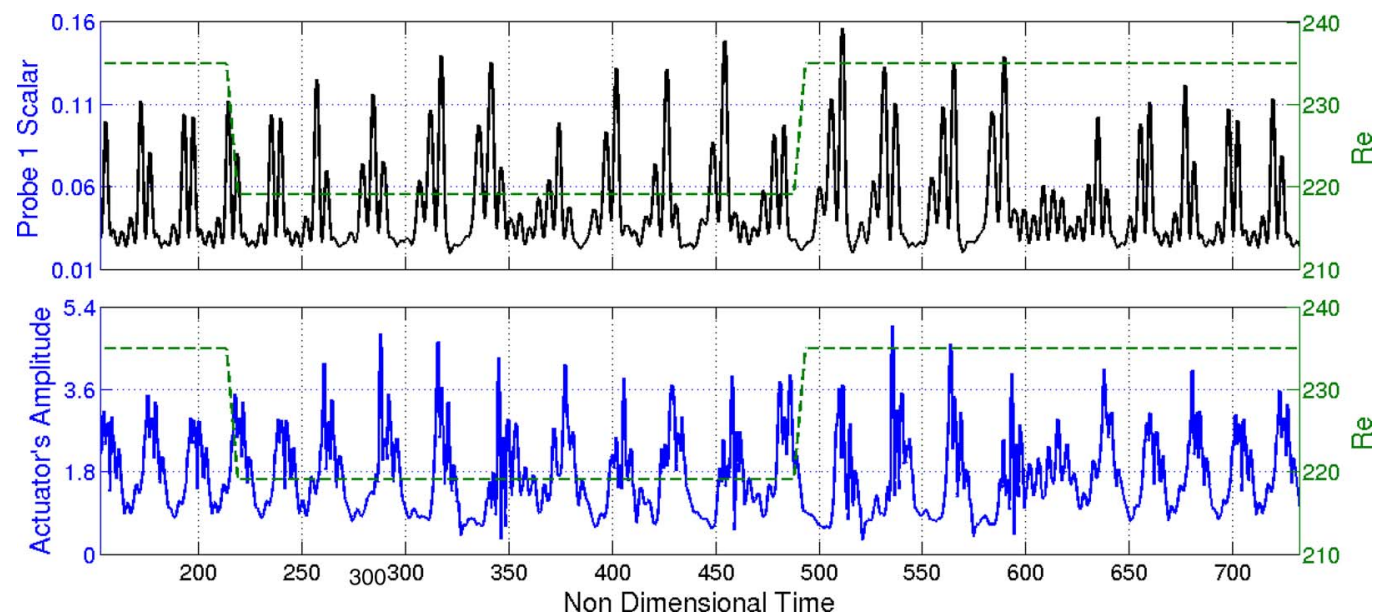

FIG. 21. Changes in the inlet condition: The Reynolds number of the controlled flow decreases from 235 to 219 and recovers its initial value following a ramp (green-dashed plot)). Upper plot: Scalar concentration at probe 1 as a function of time. Lower plot: Instantaneous amplitude of the forcing in each actuator. The control system adapts its command to stabilize the wake to the new inlet condition.

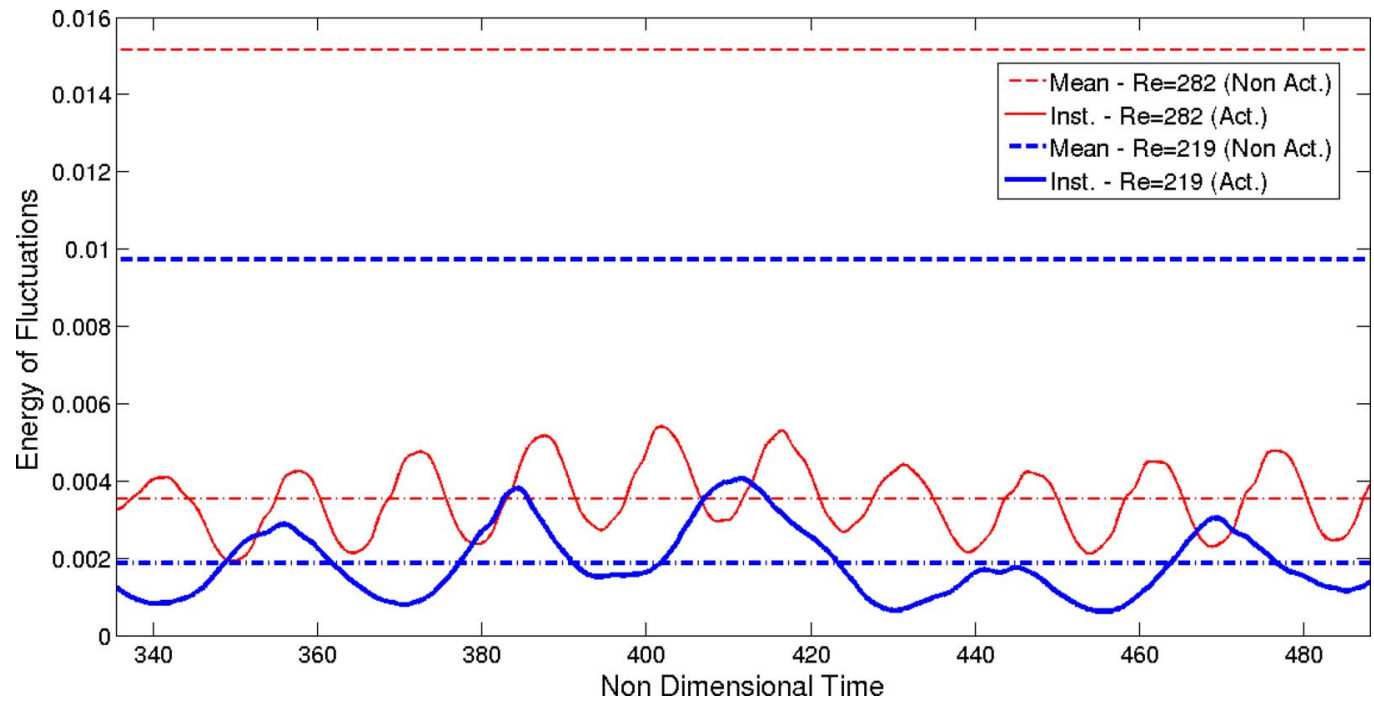

FIG. 22. Instantaneous and time averages of kinetic energy fluctuations when inlet conditions change in time as in Figs. 20 and 21.

Alteration of the inlet velocity was also considered with changes up to $20 \%$ in the Reynolds number. For this purpose we considered a time varying velocity at the inlet following an ascending (resp., descending) ramp, plateaued at the new value and then decreased (resp., increased) to the original one. We can observe in Figures 20 and 21 that the controller is able to adapt the command and preserve the control performance. After a transient, the control system stabilizes the cylinder wake under the new conditions and the fluctuating energy diminishes (Fig. 22). Note that this is a non-trivial proof of robustness since the frequency of the flow is altered as a consequence of the change in the inlet velocity.

\section{CONCLUSIONS}

A novel streakline-based closed-loop control methodology was developed and tested in a numerical simulation. The approach we propose is based on images of the flow and essentially consists 
in preventing the flow field to exhibit some undesirable patterns. A laminar flow around a circular cylinder was chosen to demonstrate the algorithm performance in reducing the wake oscillations amplitude. The control was achieved via plasma actuators disposed on the cylinder surface that add momentum tangentially to the cylinder surface close to the boundary layer separation line. The control system is based on an ARX system identification model with a Generalized Predictive Controller.

The un-modelled nonlinear dynamics of the physical system may limit the flexibility in the objective and the performance of the control based on a single or very few probes. The present image-based system effectively relies on the use of a large number of non-intrusive sensors. The resulting linear controller was shown to achieve an efficient feedback control with a reduction of about $85 \%$ in the mean fluctuating kinetic energy. In the context of separated flows, the control effectiveness is often limited by the absence of workable cost functions. ${ }^{25}$ In this regard, our study represents a step forward, extending the use of linear system models in complex flows by allowing the choice of a suitable cost function. The goal of this work was restricted to obtaining a narrow wake, which, for this kind of flow, is known to produce a reduction of the kinetic energy associated with wake fluctuations. Extensions to other goals usually considered in separated flows like lift enhancement or separation reduction are possible via a suitable choice of the probes location.

Compared to noise amplifier flows, the present test case puts markedly less stringent constrains on how to take into account the effects of external disturbances in the control strategy. The robustness of the control system was tested by considering pseudo-random noise in the measures of the sensors, tracer injection system and uniformity of the inlet flow. We also tested the ability of the controller to adjust to a new inlet velocity. In all these cases, and within the range of the parameters studied, we obtained satisfactory results. These results provide confidence in application of this approach to physical experiments that may be close to field configurations. The number of probes required depends on the characteristics of the flow considered and the tuning of parameters may be laborious in some cases. In physical experiments, the use of a numerical benchmark to determine the appropriate parameters may simplify this procedure. Compared with other control techniques relying on images of the flow, crucial advantages of the present implementation are the simplicity of the instrumentation required and a reduction of the post-processing steps of the acquired images. Further, it allows to handle situations where probes cannot be used (such as a high temperature) or would be too intrusive. The proposed control methodology is generic enough to be adapted to other experiments, in particular those involving larger Reynolds number and three-dimensional flows where the tracer concentration can be monitored with images acquired from two or more cameras suitably disposed.

The present work can be considered as a proof-of-concept and an actual application to a realworld problem may benefit from the use of a more robust ARMAX model instead of the present ARX implementation. Mutatis mutandis, the corresponding control methodology is quite similar and efforts towards application of the present methodology to a real application are currently in progress.

\section{ACKNOWLEDGMENTS}

We thank C. Collewet for helpful comments. This research is supported by grants of the UBA, CONICET, and CNRS.

${ }^{1}$ J. Lin, "Review of research on low-profile vortex generators to control boundary-layer separation," Prog. Aerosp. Sci. 38, 389-420 (2002).

${ }^{2}$ G. Godard and M. Stanislas, "Control of a decelerating boundary layer. Part 3: Optimization of round jets vortex generators," Aerosp. Sci. Technol. 10, 455-464 (2006).

${ }^{3}$ T. Duriez, J. Aider, and J. Wesfreid, "Self-sustaining process through streak generation in a flat-plate boundary layer," Phys. Rev. Lett. 103, 144502 (2009).

${ }^{4}$ A. Roshko, "On the wake and drag of bluff bodies," J. Aeronaut. Sci. 22, 124-132 (1955).

${ }^{5}$ J. Kostas, J. Foucaut, and M. Stanislas, "The flow structure produced by pulsed-jet vortex generators in a turbulent boundary layer in an adverse pressure gradient," Flow, Turbul. Combust. 78, 331-363 (2007).

${ }^{6}$ B. Thiria, S. Goujon-Durand, and J. Wesfreid, "The wake of a cylinder performing rotary oscillations," J. Fluid Mech. 560, 123-147 (2006). 
${ }^{7}$ G. Artana, R. Sosa, E. Moreau, and G. Touchard, "Control of the near wake-flow around a circular cylinder with electrohydrodynamic actuators," Exp. Fluids 35, 580-588 (2003).

${ }^{8}$ M. Munska and T. Mclaughlin, "Circular cylinder flow control using plasma actuators," AIAA Paper 2005-0141, 2005.

${ }^{9}$ T. Jukes and K. Choi, "Control of unsteady flow separation over a circular cylinder using dielectric-barrier-discharge surface plasma," Phys. Fluids 21, 094106 (2009).

${ }^{10}$ X. Ma, G. Karamanos, and G. Karniadakis, "Dynamics and low-dimensionality of a turbulent near wake,” J. Fluid Mech. 410, 29-65 (2000).

${ }^{11}$ H. Aling, S. Banerjee, A. K. Bangia, V. Cole, J. L. Ebert, A. Emami-Naeini, K. F. Jensen, I. G. Kevrekidis, and S. Shvartsman, "Nonlinear model reduction for simulation and control of rapid thermal processing," Proc. Am. Control Conf. 8, 2233-2238 (1997).

${ }^{12}$ A. Deane, I. Kevrekidis, G. Karniadakis, and S. Orszag, "Low-dimensional models for complex geometry flows: Application to grooved channels and circular cylinders," Phys. Fluids 3(10), 2337 (1991).

${ }^{13}$ G. Artana, A. Cammilleri, J. Carlier, and E. Memin, "Strong and weak constraint variational assimilations for reduced order modelling for flow control,” J. Comput. Phys. 231(8), 3264-3288 (2012).

${ }^{14}$ J. D'Adamo, N. Papadakis, E. Memin, and G. Artana, "Variational assimilation of POD low-order dynamical systems," J. Turbul. 8(9), 1-22 (2007).

${ }^{15}$ J. Gerhard, M. Pastoor, R. King, B. R. Noack, A. Dillmann, M. Morzyński, and G. Tadmor, "Model-based control of vortex shedding using low-dimensional Galerkin models," in 33rd AIAA Fluids Conference and Exhibit, Orlando, FL, USA, 23-26 June 2003, AIAA Paper 2003-4262.

${ }^{16}$ B. R. Noack, G. Tadmor, and M. Morzyński, "Actuation models and dissipative control in empirical Galerkin models of fluid flows," in Proceedings of the 2004 American Control Conference, Boston, MA, USA, 30 June-2 July (IEEE, Piscataway, NJ, 2004), Vol. 6, pp. 5722-5727, paper FrP15.6.

${ }^{17}$ B. Protas, "Linear feedback stabilization of laminar vortex shedding based on a point vortex model," Phys. Fluids 16(12), 4473-4488 (2004).

${ }^{18} \mathrm{~V}$. Kalb and A. Deane, "An intrinsic stabilization scheme for proper orthogonal decomposition based low-dimensional models," Phys. Fluids 19(5), 054106 (2007).

${ }^{19}$ M. Bergmann and L. Cordier, "Optimal control of the cylinder wake in the laminar regime by trust-region methods and pod reduced-order models,” J. Comput. Phys. 227, 7813-7840 (2008).

${ }^{20}$ M. Pastoor, L. Henning, B. R. Noack, R. King, and G. Tadmor, "Feedback shear layer control for bluff body drag reduction," J. Fluid Mech. 608, 161-196 (2008)

${ }^{21}$ K. Aleksic, R. Luchtenburg, D. King, B. R. Noack, and J. Pfeiffer, "Robust nonlinear control versus linear model predictive control of a bluff body wake," in 5th Flow Control Conference, 28 June-1 July 2010, Chicago Illinois, AIAA Paper 2010-4833.

${ }^{22}$ J.-N. Juang and M. Phan, "Deadbeat predictive controllers," Technical Memorandum 112862 (NASA, 1997).

${ }^{23}$ M. A. Kegerise, R. H. Cabell, and L. N. Cattafesta, "Real time feedback control of flow-induced cavity tones - Part 1: Fixed-gain control," J. Sound Vib. 307, 906-923 (2007).

${ }^{24}$ M. A. Kegerise, R. H. Cambell, and L. N. Cattafesta, "Real time feedback control of flow-induced cavity tones - Part 2: Adaptive control," J. Sound Vib. 307, 924-940 (2007).

${ }^{25}$ S.-C. Huang and J. Kim, "Control and system identification of a separated flow," Phys. Fluids 20, 101509 (2008).

${ }^{26}$ A. Herve, D. Sipp, P. J. Schmid, and M. Samuelides, "A physics-based approach to flow control using system identification," J. Fluid Mech. 702, 26-58 (2012).

${ }^{27}$ K. Shariff, T. H. Pulliam, and J. Ottino, "A dynamical systems analysis of kinematics in the time-periodic wake of a circular cylinder," Lectures in Applied Mathematics (American Mathematical Society, 1991), Vol. 28, pp. 613-646.

${ }^{28}$ E. Ziemniak, C. Jung, and T. Tel, "Tracer dynamics in open hydrodynamical flows as chaotic scattering," Physica D76, 123-146 (1994).

${ }^{29}$ G. Haller, "Exact theory of unsteady separation for two-dimensional flows," J. Fluid Mech. 512, 257 (1994).

${ }^{30}$ M. Alam, W. Liu, and G. Haller, "Closed-loop separation control: an analytical approach,” Phys. Fluids 18, 043601 (2006).

${ }^{31}$ Y. Wang, G. Haller, A. Banaszuk, and G. Tadmor, "Closed-loop Lagrangian separation control in a bluff body shear flow model," Phys. Fluids 15, 2251-2256 (2003).

${ }^{32}$ R. T. Fomena and C. Collewet, "Fluid flow control: a vision-based approach," Int. J. Flow Control 3, 133-169 (2011).

${ }^{33}$ H. Yu, M. Leeser, G. Tadmor, and S. Siegel, "Real-time particle image velocimetry for feedback loops using FPGA implementation," J. Aerosp. Comput., Inf. Commun. 3, 52-62 (2006).

${ }^{34}$ N. Gautier and J. L. Aider, "Control of the flow behind a backward-facing step by visual feedback," preprint arXiv: 1306.4554 (2013).

${ }^{35}$ J. D'Adamo, L. M. Gonzalez, A. Gronskis, and G. Artana, "The scenario of two-dimensional instabilities of the cylinder wake under electrohydrodynamic forcing: a linear stability analysis," Fluid Dyn. Res. 44, 20 (2012).

${ }^{36}$ F. Archambeau, N. Méchitoua, and M. Sakiz, "Code Saturne: a finite volume code for the computation of turbulent incompressible flows," Int. J. Finite Vol. 1, 1-62 (2004).

${ }^{37}$ J. R. Roth, D. M. Sherman, and S. P. Wilkinson, "Electrohydrodynamic flow control with a glow-discharge surface plasma," AIAA J. 38, 1166-1172 (2000).

${ }^{38}$ A. Gronskis, "Modelos numericos para el estudio de flujos externos controlados con actuadores EHD," Ph.D. thesis (University of Buenos Aires, 2009).

${ }^{39}$ L. Ljung, System Identification: Theory for the User (Prentice-Hall Inc., Englewood Cliffs, NJ, 1987).

${ }^{40}$ G. Tadmor, O. Lehmann, B. R. Noack, and M. Morzynski, "Mean field representation of the natural and actuated cylinder wake," Phys. Fluids 22, 034102 (2010).

${ }^{41}$ E. Gillies, "Low-dimensional control of the circular cylinder wake," J. Fluid Mech. 371, 157-178 (1998).

${ }^{42}$ K. Cohen, S. Aradag, S. Siegel, J. Seidel, and T. McLaughlin, Low Reynolds Number Aerodynamics and Transition, edited by M. Serdar Genc (InTech, Europe, 2012), Chap. 6, pp. 117-138. 
${ }^{43}$ L. N. Cattafesta, Q. Song, D. R. Williams, C. W. Rowley, and F. S. Alvi, “Active control of flow-induced cavity oscillations," Prog. Aerosp. Sci. 44, 479-502 (2008).

${ }^{44}$ K. Roussopoulos, "Feedback control of vortex shedding at low Reynolds number," J. Fluid Mech. 248, 267-296 (1993).

${ }^{45}$ N. E. Huang and S. Shen, Hilbert-Huang Transform and Its Applications (World Scientific Publishing, Singapore, 2005).

${ }^{46}$ F. L. Lewis and V. L. Syrmos, Optimal Control (John Wiley \& Sons, Inc., 1995). 